\title{
Efficient Anti-Glioma Therapy Through the Brain-Targeted RVG I5-Modified Liposomes Loading Paclitaxel-Cholesterol Complex
}

\author{
Xin Xin (iD) \\ Wei Liu \\ Zhe-Ao Zhang \\ Ying Han \\ Ling-Ling Qi \\ Ying-Ying Zhang \\ Xin-Tong Zhang \\ Hong-Xia Duan \\ Li-Qing Chen \\ Ming-Ji Jin \\ Qi-Ming Wang \\ Zhong-Gao Gao (D) \\ Wei Huang
}

State Key Laboratory of Bioactive Substance and Function of Natural Medicines, Department of Pharmaceutics, Institute of Materia Medica, Chinese Academy of Medical Sciences and Peking Union Medical College, Beijing, 100050,

People's Republic of China
Correspondence: Zhong-Gao Gao; Wei Huang

State Key Laboratory of Bioactive

Substance and Function of Natural

Medicines, Institute of Materia Medica,

Chinese Academy of Medical Sciences and

Peking Union Medical College, I Xian

Nong Tan Street, Beijing, 100050, People's

Republic of China

Tel +86-10-63026505

Email zggao@imm.ac.cn;

huangwei@imm.ac.cn
Background: Glioma is the most common primary malignant brain tumor with a dreadful overall survival and high mortality. One of the most difficult challenges in clinical treatment is that most drugs hardly pass through the blood-brain barrier (BBB) and achieve efficient accumulation at tumor sites. Thus, to circumvent this hurdle, developing an effectively traversing BBB drug delivery nanovehicle is of significant clinical importance. Rabies virus glycoprotein (RVG) is a derivative peptide that can specifically bind to nicotinic acetylcholine receptor $(\mathrm{nAChR})$ widely overexpressed on $\mathrm{BBB}$ and glioma cells for the invasion of rabies virus into the brain. Inspired by this, RVG has been demonstrated to potentiate drugs across the BBB, promote the permeability, and further enhance drug tumorspecific selectivity and penetration.

Methods: Here, we used the RVG15, rescreened from the well-known RVG29, to develop a brain-targeted liposome (RVG15-Lipo) for enhanced BBB permeability and tumor-specific delivery of paclitaxel (PTX). The paclitaxel-cholesterol complex (PTX-CHO) was prepared and then actively loaded into liposomes to acquire high entrapment efficiency (EE) and fine stability. Meanwhile, physicochemical properties, in vitro and in vivo delivery efficiency and therapeutic effect were investigated thoroughly.

Results: The particle size and zeta potential of PTX-CHO-RVG15-Lipo were $128.15 \pm 1.63$ $\mathrm{nm}$ and $-15.55 \pm 0.78 \mathrm{mV}$, respectively. Compared with free PTX, PTX-CHO-RVG15-Lipo exhibited excellent targeting efficiency and safety in HBMEC and C6 cells, and better transport efficiency across the BBB in vitro model. Furthermore, PTX-CHO-RVG15-Lipo could noticeably improve the accumulation of PTX in the brain, and then promote the chemotherapeutic drugs penetration in $\mathrm{C}^{\text {luc }}$ orthotopic glioma based on in vivo imaging assays. The in vivo antitumor results indicated that PTX-CHO-RVG15-Lipo significantly inhibited glioma growth and metabasis, therefore improved survival rate of tumor-bearing mice with little adverse effect.

Conclusion: Our study demonstrated that the RVG15 was a promising brain-targeted specific ligands owing to the superior BBB penetration and tumor targeting ability. Based on the outstanding therapeutic effect both in vitro and in vivo, PTX-CHO-RVG15-Lipo was proved to be a potential delivery system for PTX to treat glioma in clinic.

Keywords: glioma, blood-brain barrier, RVG15, liposome, paclitaxel

\section{Introduction}

The incidence of brain tumors has increased over time with glioma being one of the most common primary malignants, accounting for more than $30 \%$ of central nervous system tumors. ${ }^{1,2}$ The main treatment of glioma is traditional surgery, 


\section{Graphical Abstract}

A
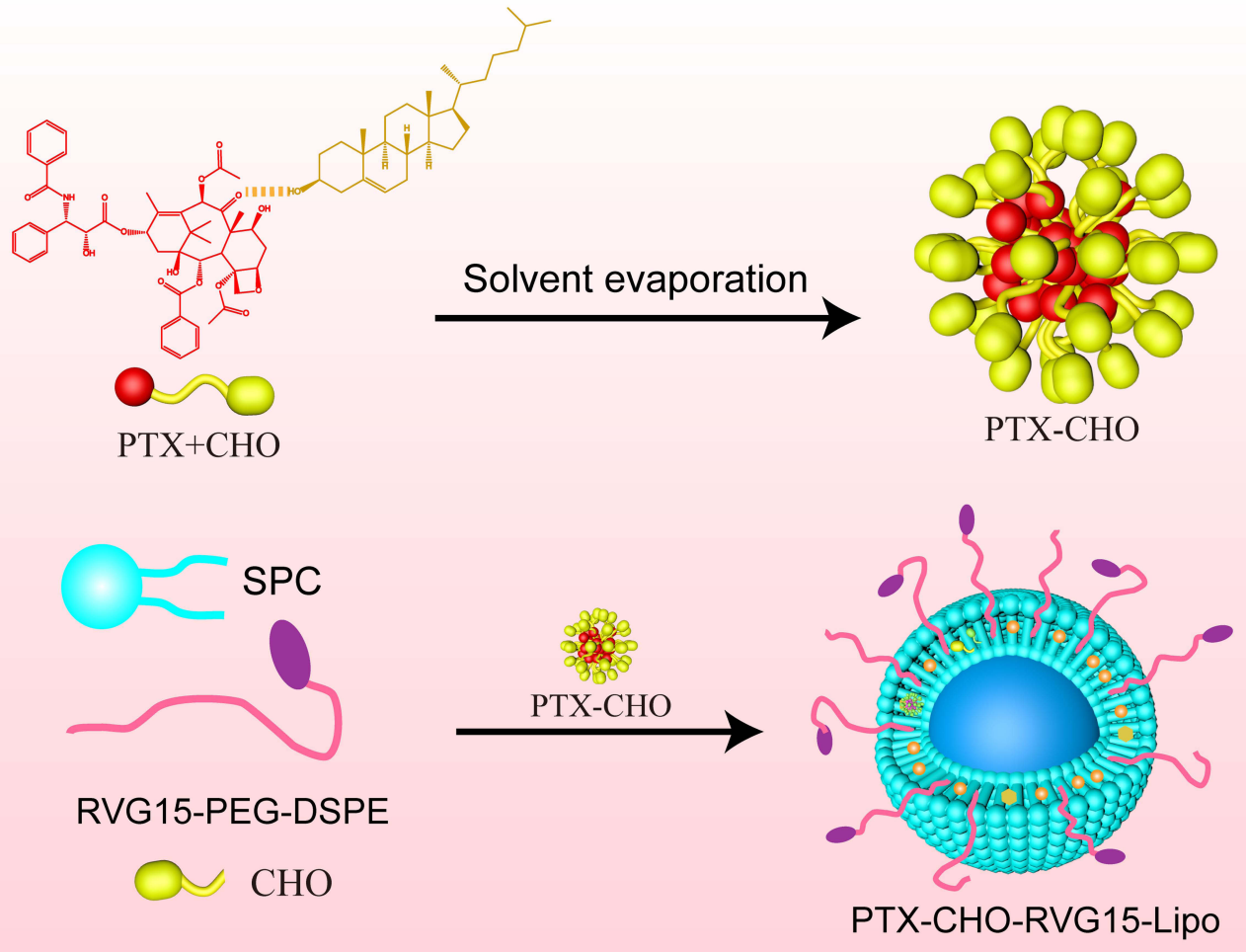

B

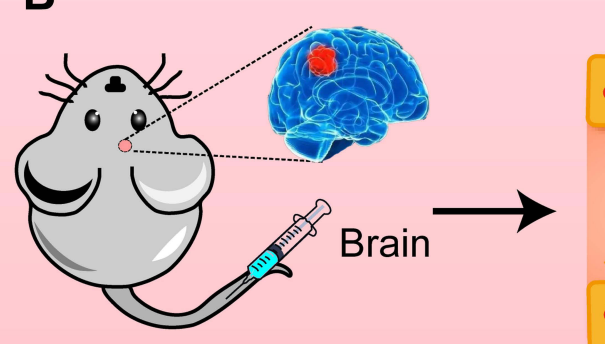

Blood-brain Barrier
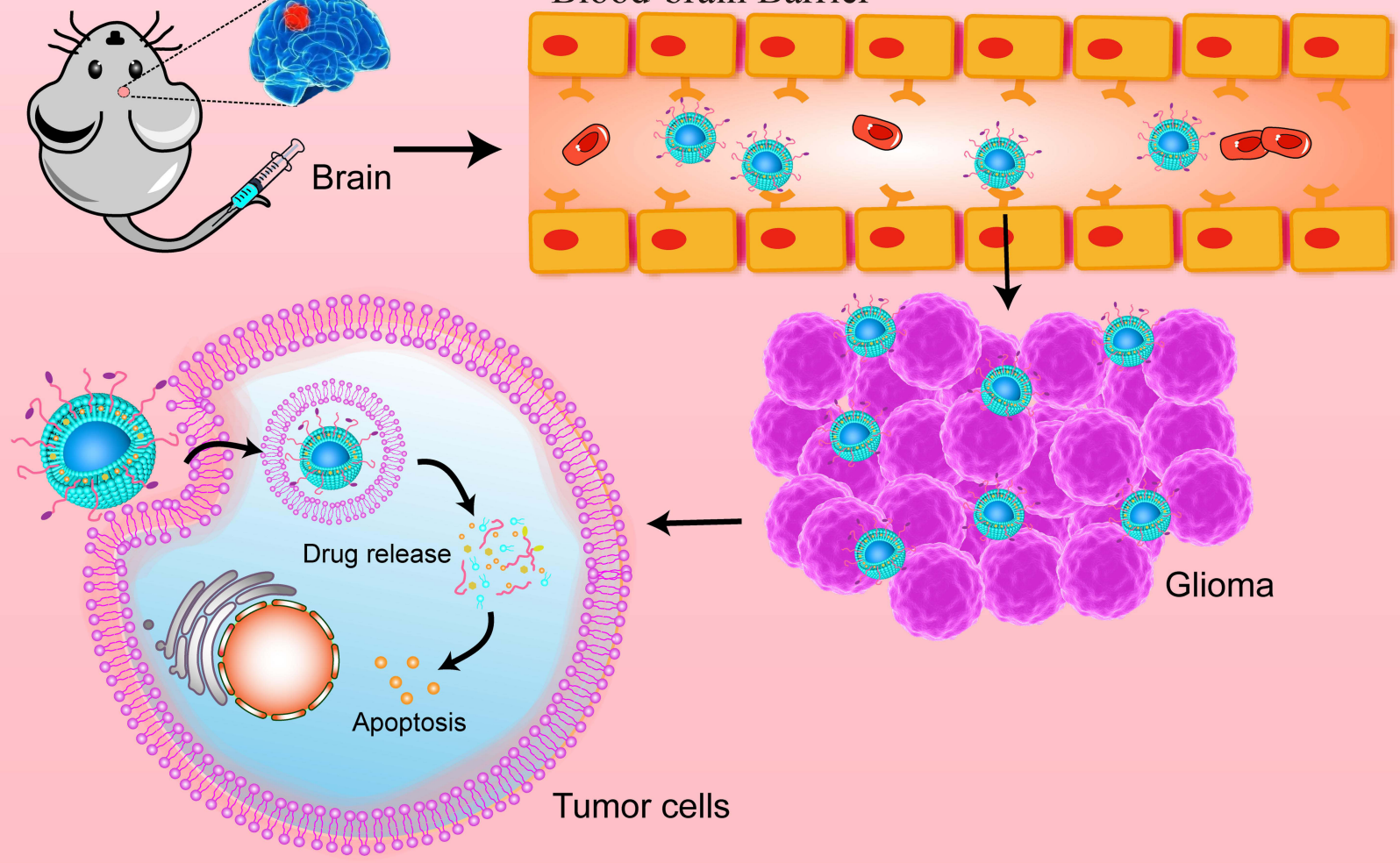
but generally glioma cannot be completely removed with high relapse rate, poor prognosis and the median survival length $<15$ months after surgery. ${ }^{3-5}$ It has become a serious threat to human health. To restrain and effectively eradicate glioma, chemotherapy becomes the most common choice. However, current chemotherapy cannot achieve the desired effect due to the presence of the blood-brain barrier (BBB) and severe side effects. ${ }^{6}$

$\mathrm{BBB}$ is a complex protective barrier between the central nervous system and peripheral blood circulation. It is a tight structure composed of brain capillary endothelial cells (BCECs), astrocyte terminal foot, peripheral cells and vascular basement membrane. Compared with other tissues, BBB has almost no intercellular space, which effectively limits the extracellular diffusion of solutes or drugs. Generally, more than $98 \%$ of small molecule drugs and almost all large molecules cannot penetrate BBB. ${ }^{7-10}$ Brain targeted drug delivery systems are designed to address these problems, which traverse the BBB and selectively improve drug concentration in brain tissues. So far, there are three main types of drug delivery systems in the brain: (1) absorptive-mediated transcytosis (AMT); (2) carrier-mediated transport (CMT) systems; and (3) receptor-mediated transcytosis (RMT). Due to the fact that BCECs overexpress many different receptors, RMT has emerged as one of the most commonly used strategies for brain targeted drug delivery. As expected, RMT demonstrated the enhanced BBB penetration of chemotherapeutic drugs and improved treatment efficacy of glioma. RMT, as a selective active transport method, has been widely used in brain targeting research. ${ }^{11-13}$

The nicotinic acetylcholine receptor (nAChR) is an ion gated channel receptor, which is mainly expressed in neuronal cells. ${ }^{14-16}$ Furthermore, there also have been many studies of nAChR expression in brain endothelial cells as well as other tumor cells, ${ }^{17,18}$ especially glioma. ${ }^{19}$ Thus, targeting nAChR can propel the drug across $\mathrm{BBB}$ and achieve tumor-specific accumulation based on the RMT effect. The rabies virus glycoprotein peptide (RVG) is a kind of glycoprotein with a 29amino acid sequence expressed on the surface of rabies virus, which is able to bind specifically to $\mathrm{nAChR}$ expressed in neuronal cells for virus entry. ${ }^{20-22}$ Especially, the $\alpha 7$ subunit of the $\mathrm{nAChR}$ is widely expressed in the brain including capillary endothelial cells. ${ }^{23}$ Although the tight junctions formed by brain endothelial cells prevent many drugs from passing through the BBB, studies have shown that the RVG29 peptide can bring therapeutic agents across the BBB and rapidly reach the brain tumor site by binding to nAChR. ${ }^{19}$ In summary, the RVG29 peptide can be used as a ligand for brain targeted drug delivery system. However, given that the high molecular weight of RVG29, the cost and particle size of the nanocarriers using RVG29 as ligands are dissatisfied. In addition, the large size nanocarriers hardly penetrate the tumor parenchyma more effectively and lead to most drugs distribution around tumor blood vessels. In view of the above facts, our lab previously rescreened the RVG29 sequence and obtained RVG15 with only 15 amino acids, of which the brain targeting efficiency was equivalent to that of RVG29 (our CN patent No. ZL201810317197.X). Then our lab constructed braintargeting micelles employing RVG15 as ligands and the results indicated that the prepared nanocarriers displayed good drug-carrying capacity across the BBB. ${ }^{24}$ RVG15 was firstly reported by our lab and employing RVG15 as a ligand to prepare small size and economic nanocarriers was conducive for achieving effective brain targeting.

Paclitaxel (PTX), as a natural broad-spectrum antineoplastic drug, is recommended as a first-line strategy against various types of cancers. ${ }^{25-27}$ However, due to the low solubility, poor BBB penetration ability and several side effects, the clinical efficacy of PTX in glioma is limited. PTX is expected to achieve better anti-tumor effects if it is able to reach glioma tumor sites at sufficient concentration. Therefore, the development of a drug delivery system that can significantly improve the BBB permeability and glioma aggregation of PTX has high clinical application value.

In this study, we have developed a brain-targeted drug delivery system based on RVG15 modified liposome (RVG15-Lipo). To acquire high drug loading and encapsulation rate, paclitaxel-cholesterol complex (PTX-CHO) was firstly prepared and then loaded into the phospholipid bilayer of liposome. Due to the high binding affinity to $\mathrm{nAChR}$, the modified RVG15 improve the BBB penetrating capability of drug and the anti-glioma efficacy (as illustrated in Figure 1). We synthesized the targeted materials, prepared and characterized the liposomes and evaluated the BBB crossing and anti-glioma potential of RVG15-modified liposomes both in vitro and in vivo. 
A
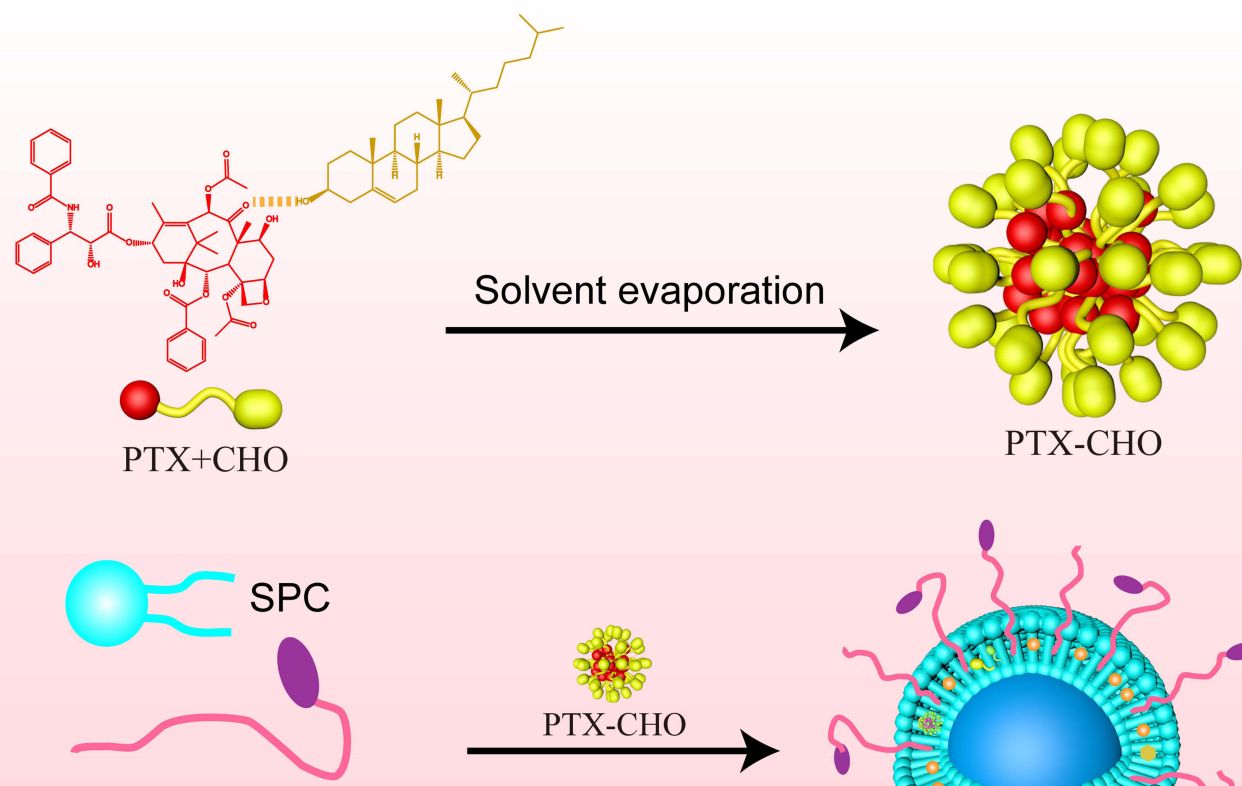

RVG15-PEG-DSPE

CHO

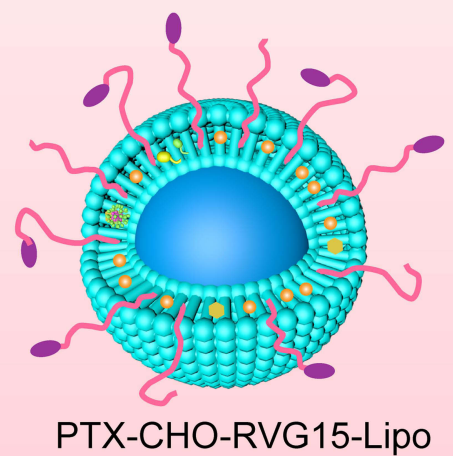

B

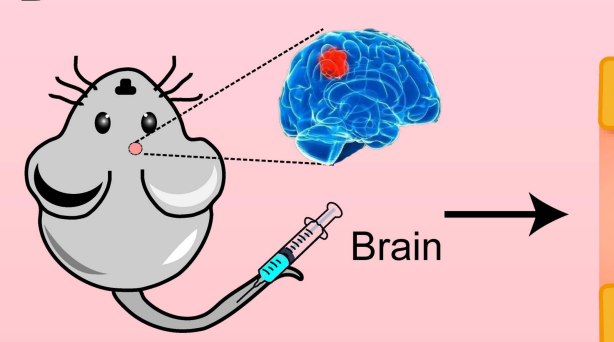

Blood-brain Barrier
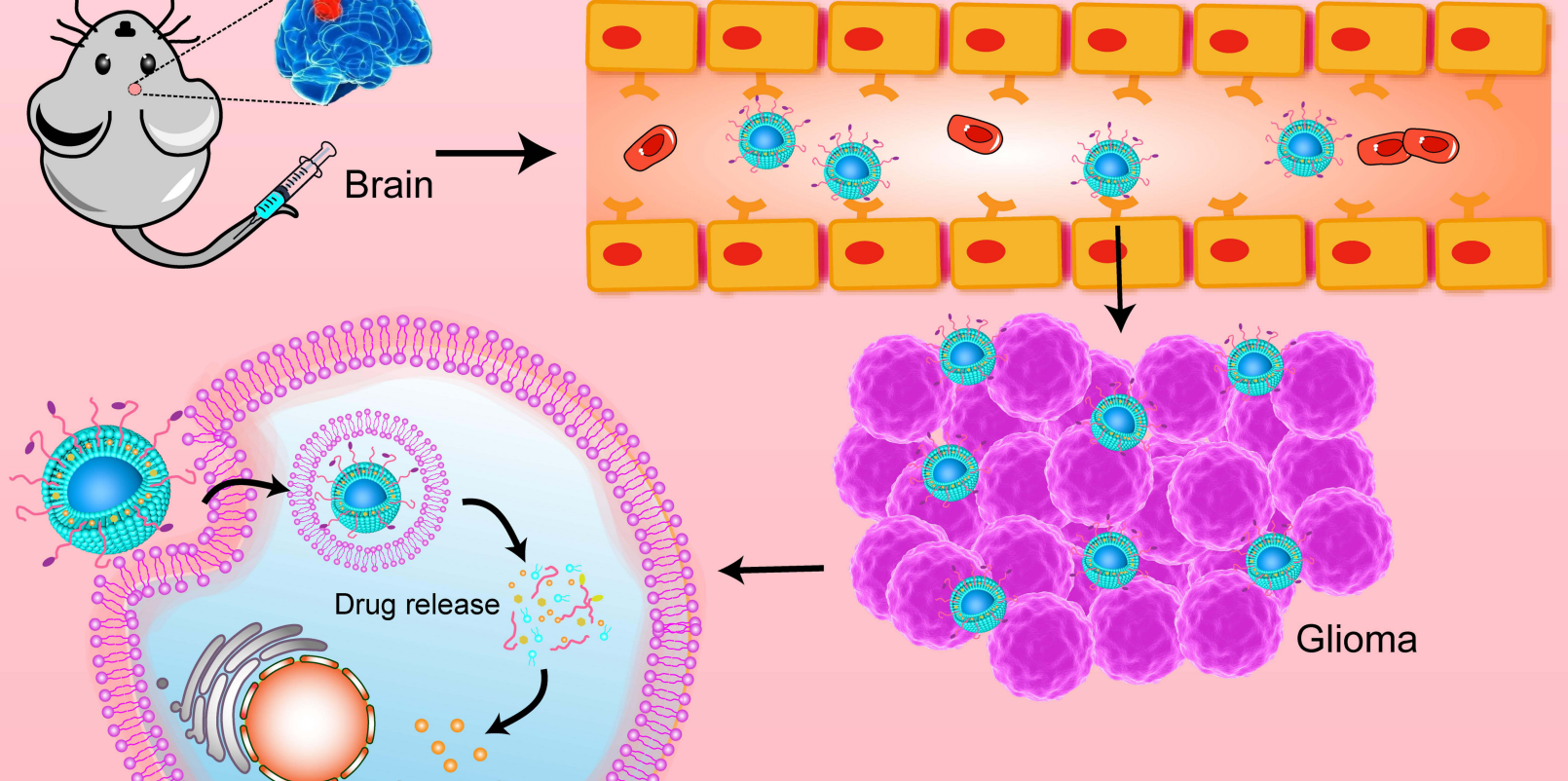

Apoptosis

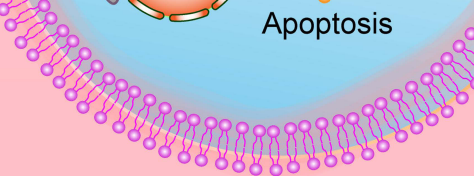

\section{Tumor cells}

Figure I Schematic illustration of PTX-CHO-RVG I5-Lipo for highly effective and specific anti-glioma chemotherapy.

Notes: (A) Assembly processes of PTX-CHO and PTX-CHO-RVGI5-Lipo. (B) PTX-CHO-RVG I5-Lipo could penetrate the blood-brain barrier and deliver paclitaxel into glioma tissue in vivo selectively though specifically binding to nicotinic acetylcholine receptors expressed on the brain capillary endothelial cells and the glioma cells. Abbreviations: PTX-CHO, paclitaxel-cholesterol complex; PTX-CHO-RVGI5-Lipo, PTX-CHO-loaded RVGI5-modified liposomes. 


\section{Materials and Methods}

\section{Materials}

PTX was bought from Dalian Meilun Biotechnology Co., Ltd. (Dalian, P.R. China). The peptide RVG15Cys (YTIWCDIFTNSRGKRC) was synthesized by GL Biochem (Shanghai) Ltd. (Shanghai, P.R. China). Soybean phosphatidylcholine (SPC), cholesterol, DSPE-mPEG $_{2000}$ (Mw 2794.07 Da) and DSPE$\mathrm{PEG}_{2000}$-maleimide (DSPE-PEG ${ }_{2000}$-Mal, Mw 2875 $\mathrm{Da}$ were commercially obtained from AVT (Shanghai) Pharmaceutical Co., Ltd (Shanghai, P.R. China). Sephadex G-50 was supplied by Solarbio (Shanghai, P.R. China). Anhydrous ethanol, acetonitrile, and methanol (HPLC grade) were purchased from Sigma-Aldrich Co. (St Louis, MO, USA).

Fetal bovine serum (FBS) and $0.25 \%$ trypsin $0.53 \mathrm{mM}$ ethylenediaminetetraacetic acid solution were obtained from Thermo Fisher Scientific Co., Ltd. (Beijing, P.R. China). Dulbecco modified Eagle medium (DMEM), Roswell Park Memorial Institute-1640 (RPMI-1640) medium, and phosphate-buffered saline (PBS) were purchased from GE Healthcare (Boston, MA, USA). Fluorescein isothiocyanate/propidium iodide (FITC/PI) apoptosis detection kit and Cell Counting Kit-8 (CCK-8) were bought from Dojindo Laboratories (Kumamoto, Japan). DNA content quantitation assay (cell cycle) was supplied by Solarbio (Shanghai, P.R. China). 1.1-dioctadecyltetramethyl indotricarbocyanine iodide (DiR) was commercially obtained from Biotium Inc. (Hayward, CA, USA). Coumarin-6 and Hoechst 33258 were obtained from Sigma-Aldrich Co. (St Louis, MO, USA). All of the other chemicals were of analytical purity and used without further purification.

\section{Synthesis and Characterization of RVG I5- $\mathrm{PEG}_{2000}$-DSPE}

RVG15-PEG 2000 -DSPE was synthesized by conjugating the cysteine residue of RVG15-Cys to DSPE-PEG ${ }_{2000^{-}}$ Mal using a previously reported method. ${ }^{28}$ Briefly, RVG15-Cys and DSPE-PEG 2000 -Mal (at a molar ratio of 1.5:1) were dissolved in $\mathrm{pH} 8.0$ HEPEs buffer, and then filled with nitrogen for protection. The reaction was carried out for $16 \mathrm{~h}$ at ambient temperature under moderate stirring. The resulting reaction mixture was isolated through dialysis (MWCO 12000) for $24 \mathrm{~h}$ against free
RVG15. The final product was obtained via cryodesiccation. Synthesis of RVG15-PEG ${ }_{2000}$-DSPE was confirmed by ${ }^{1} \mathrm{H}$ NMR spectroscopy (400 MHz, Varian Medical Systems, Inc., Palo Alto, CA, USA) and further verified by matrix-assisted laser desorption/ionization time of flight mass spectrometry (MALDI-TOF-MS) (4800 Plus, Applied Biosystems Inc., Waltham, MA, USA). For MALDI-TOF-MS analysis, the detection matrix was 3-indoleacetic acid.

\section{Preparation of Paclitaxel-Cholesterol Complex}

Paclitaxel-cholesterol complex (PTX-CHO complex) was prepared by solvent evaporation method. ${ }^{29}$ Briefly, PTX and cholesterol (molar ratio 1:1) were dissolved in appropriate amount of acetone and gently stirred for $2 \mathrm{~h}$ at 50 ${ }^{\circ} \mathrm{C}$. Acetone was removed by rotary evaporation and vacuum drying to obtain PTX-CHO complex. Compared with PTX, cholesterol and physical mixture of PTX and cholesterol, the structure of PTX-CHO complex was confirmed using a differential scanning calorimetry (DSC) Analysis (TA Instruments Inc., Sherman, TX, USA).

\section{Preparation of PTX-CHO Complex Loaded Liposomes with or without RVGI5 Modification}

Liposomes for this study were prepared using thin film hydration method as previously reported by our laboratory. ${ }^{30}$ Lipid compositions of different liposomes were as follows: (a) PTX-CHO-loaded PEGylated liposomes (PTX-CHO-Lipo), SPC/cholesterol/DSPE$\mathrm{mPEG}_{2000}$ (molar ratio $=40: 4: 2$ ), drug-lipid ratio was 1:50; (b) PTX-CHO-loaded liposomes with RVG15 modification (PTX-CHO-RVG15-Lipo), SPC/cholesterol/DSPE$\mathrm{mPEG}_{2000} / \mathrm{RVG}$-PEG-DSPE (molar ratio $=40: 4: 1: 1$ ), drug-lipid ratio was 1:50. Prescribed amount of PTX-CHO complex was added directly in chloroform containing all lipid materials and dissolved completely by ultrasound. A lipid film was formed after chloroform removed by rotary evaporation at $40^{\circ} \mathrm{C}$ for $40 \mathrm{~min}$ and further drying in vacuum. Finally, the film was hydrated with $5 \%$ glucose solution, and subjected to sonicated for $15 \mathrm{~min}$ (power: 50 $\mathrm{W}$, ultrasonic 2 seconds, intermittent 1 second) under an ultrasonic cell pulverizer (Scientz 950E; Ningbo Scientz Biotechnology Co., Ltd., Zhejiang, P.R. China). 
Coumarin-6-labeled and DiR-labeled liposomes were prepared by respectively adding appropriate amount of coumarin-6 or DiR to the lipid solution, replacing PTXCHO complex. The blank liposome was prepared in the same method without the drug.

\section{Characterization}

The mean particle size, polydispersity index (PDI), and zeta potential of PTX-CHO-RVG15-Lipo were measured by dynamic light scattering (DLS) and electrophoretic light scattering (Zetasizer Nano ZS90; Malvern Instruments Ltd., UK). The morphology of PTX-CHORVG15-Lipo was characterized by negative staining method using a transmission electron microscope (TEM, JEM-1400PLUS, JEOL Ltd., Tokyo, Japan).

The minicolumn centrifugation technique as previously reported $^{31}$ was used to separate free PTX from prepared liposomes, and the entrapment efficiency (EE\%) and drug loading capacity (DL\%) of PTX-CHO-RVG15-Lipo were determined by high performance liquid chromatography (HPLC, Agilent 1260 infinity; Agilent Technologies, Santa Clara, CA, USA). The EE\% and DL\% of PTX in the liposomes were calculated as follows:

$$
\begin{gathered}
\mathrm{EE} \%=\frac{\text { Weight of encapsulated drug }}{\text { Weight of total drug }} \times 100 \% \\
\mathrm{DL} \%=\frac{\text { Weight of encapsulated drug }}{\text { Weight of total drug and lipid }} \times 100 \%
\end{gathered}
$$

The in vitro PTX release from PTX-CHO-RVG15Lipo and free PTX in a simulated physiological environment were evaluated using the dialysis method. In brief, PBS (pH 7.4) containing $0.5 \%(\mathrm{v} / \mathrm{v})$ Tween 80 was used as the release media. About $0.5 \mathrm{~mL}$ of PTX-CHO-RVG15 -Lipo solution and PTX solution were placed into dialysis bags (MW cutoff: $12000 \mathrm{Da}$ ), and then immersed in $30 \mathrm{~mL}$ release media and incubated at $37^{\circ} \mathrm{C}$ with gently shaking at $100 \mathrm{rpm}$ for $72 \mathrm{~h}$. At the predetermined time points, $0.5 \mathrm{~mL}$ of release media was sampled and replaced with equal volume of fresh release media. The obtained samples were analyzed by HPLC to measure PTX concentration and further calculate the accumulative release of PTX.

\section{Cell Line and Cell Culture}

Rat C6 glioma cell line and Human brain microvascular endothelial cell line HBMEC were purchased from the
Cell Culture Center of Institute of Basic Medical Sciences in Chinese Academy of Medical Sciences (CAMS, P.R. China). Another rat glioma cell line C6 ${ }^{\text {luc }}$ was self-constructed by our lab and could stably expressed the firefly luciferase. The $\mathrm{C} 6$ and $\mathrm{C} 6^{\text {luc }}$ cell lines were cultured in Roswell Park Memorial Institute1640 (RPMI-1640) medium supplemented with $10 \%$ (v/ v) fetal bovine serum (FBS) and $1 \%(\mathrm{v} / \mathrm{v})$ penicillinstreptomycin (Pen-Strep) in a humidified 5\% $\mathrm{CO}_{2}$ atmosphere at $37{ }^{\circ} \mathrm{C}$. The HBMEC cell line was cultured in Dulbecco modified Eagle medium (DMEM) with 10\% FBS and 1\% Pen-Strep under the same condition. The cells for the following cell experiments were in the logarithmic growth phase.

\section{Cellular Uptake Analysis Cellular Uptake on Different Cells}

Coumarin-6-labeled liposomes with or without modified RVG15 (Cou-6-RVG15-Lipo and Cou-6-Lipo) were prepared to visualize and evaluate cellular uptake and localization of liposomes. HBMEC cells and C6 cells were plated on round glass coverslips at the bottom of twelve-well plates at a density of $15 \times 10^{4}$ cells/well and cultured under $37^{\circ} \mathrm{C}$ for $24 \mathrm{~h}$. Different liposomes and free Cou- 6 were added into the plates at a Cou- 6 concentration of $5 \mu \mathrm{g} / \mathrm{mL}$. After incubation for $15,60,120$, and $240 \mathrm{~min}$ at $37^{\circ} \mathrm{C}$, the cells were washed three times with cold PBS buffer and fixed with $4 \%$ paraformaldehyde. Then DAPI was added for nuclei staining. Finally, the cells were imaged by a confocal laser scanning microscope (CLSM, Carl Zeiss LSM 710; Carl Zeiss Microscopy, Jena, Germany).

The uptake efficiency of HBMEC cells and C6 cells treated with free Cou-6, Cou-6-RVG15-Lipo and Cou6-Lipo quantitatively were analyzed using FACSCalibur flow cytometer (Becton Dickinson, Franklin Lakes, NJ, USA). Briefly, HBMEC cells and C6 cells were seeded in 12 -well plates at a density of $15 \times 10^{4}$ cells/well and cultured for $24 \mathrm{~h}$. Subsequently, the cells were incubated with free Cou-6, Cou-6-RVG15-Lipo or Cou-6-Lipo (1 $\mu \mathrm{g} /$ $\mathrm{mL}$ ) in serum-free medium for $15,60,120$, and $240 \mathrm{~min}$ at $37^{\circ} \mathrm{C}$. Then the cells were washed three times with cold PBS buffer, digested with $0.25 \%$ trypsin, centrifuged and resuspended in $0.5 \mathrm{~mL}$ PBS. The fluorescent intensity of cells was finally determined by aFACSCalibur flow cytometer. 


\section{Uptake Mechanism Study}

To study the uptake pathways of Cou-6-RVG15-Lipo for C6 cells, several membrane entry inhibitors were measured. The cells were seeded in 12-well plates with $15 \times 10^{4}$ cells/well. Subsequently, the cells were cultured with various endocytosis inhibitors, including RPMI 1640 (control), chlorpromazine hydrochloride (CPZ, 10 $\mu \mathrm{g} / \mathrm{mL})$, colchicine (Col, $4 \mu \mathrm{g} / \mathrm{mL})$, methyl- $\beta$ cyclodextrin $(M-\beta-C D, 5 \mathrm{mg} / \mathrm{mL}$ ) for $1 \mathrm{~h}$, respectively. Then the cells were exposed to serum-free medium containing $1 \mu \mathrm{g} / \mathrm{mL}$ Cou-6-RVG15-Lipo. After 2 $\mathrm{h}$ incubation, the cells were washed three times with cold PBS, trypsinized, collected and analyzed by a FACSCalibur flow cytometer.

\section{Cell Viability Assay}

CCK-8 assays were performed to evaluate the cytotoxicity of blank liposomes on HBMEC cells and C6 cells. In brief, HBMEC cells and C6 cells were respectively seeded into 96-well plates at a density of $3 \times 10^{3}$ cells/well and incubated for $24 \mathrm{~h}$ at $37{ }^{\circ} \mathrm{C}$. Then the cells were treated and cultured in fresh medium containing serial concentrations of blank RVG15-Lipo for 24 or $48 \mathrm{~h}$. The blank group was cultured with medium alone as control. According to CCK-8 kit instructions, CCK-8 reagent was added to each well and the plates were incubated for another 2 h. Finally, the plates were measured at $450 \mathrm{~nm}$ using a Synergy H1 Microplate Reader (BioTek, Dallas, TX, USA).

CCK-8 assay was used to determine cell cytotoxicity of PTX-CHO-RVG15-Lipo, PTX-CHO-Lipo and free PTX (ethanol-cremophor ELP mixture, $\mathrm{v} / \mathrm{v}=1: 1$ ) on C6 cells. Cells of the same concentration were plated. PTX-CHORVG15-Lipo, PTX-CHO-Lipo and free PTX were added at concentrations ranging from $0.01 \mu \mathrm{g} / \mathrm{mL}-50 \mu \mathrm{g} / \mathrm{mL}$, and the cells were cultured for 24 or $48 \mathrm{~h}$ at $37{ }^{\circ} \mathrm{C}$. After being treated with CCK- 8 reagent, the OD values were detected. The cells treated with medium were served as controls. Cell viability was calculated as follows.

$$
\text { Cell viability }(\%)=\frac{\text { OD test }- \text { OD blank }}{\text { OD control }- \text { OD blank }} \times 100
$$

\section{BBB Model Permeability Study}

The in vitro BBB model was built using previously reported methods to evaluate the penetrating and targeting effects of free PTX, PTX-CHO-Lipo and PTX-CHORVG15-Lipo. First, HBMEC cells were seeded on the upper side of 12-well Transwell inserts $(0.4 \mu \mathrm{m}$ pore size, Corning Co., Corning, NY, USA) at a density of $1 \times 10^{6}$ cells/insert in $0.5 \mathrm{~mL} \quad 10 \%$ FBS DMEM medium. ${ }^{32}$ Meanwhile, the lower side of the inserts was filled with $1.5 \mathrm{~mL}$ of the medium. The culture medium was refreshed every 2 days. The integrity of the HBMEC monolayers was monitored by measuring the transepithelial electrical resistance (TEER) using a TEER instrument (Millicell-ERS-2, Millipore). When the TEER value exceeded $200 \Omega \cdot \mathrm{cm}^{2}$, the monolayers were selected for the transport studies. ${ }^{33}$

The quantitative transport of the PTX-CHO-RVG15Lipo in the BBB model was measured on the monolayers described above. In Brief, FBS-free DMEM containing free PTX, PTX-CHO-Lipo and PTX-CHO-RVG15-Lipo at a PTX concentration of $100 \mu \mathrm{g} / \mathrm{mL}$ were added into the upper sides of the inserts, followed by incubation at 37 ${ }^{\circ} \mathrm{C}$ and $5 \% \mathrm{CO}_{2}$. Then $300 \mu \mathrm{L}$ of medium in the lower side was removed at 60, 90, 120, 150 and $180 \mathrm{~min}$, and replaced with an equal volume of fresh medium. The transport ratios (\%) of different agents were calculated using the above established HPLC method.

\section{Cell Apoptosis Assay}

Hoechst staining assay was used to analyze cell apoptosis qualitatively. C6 cells were seeded into 12-well plates and cultured for $24 \mathrm{~h}$. Then the culture medium was replaced with fresh medium containing $1 \mu \mathrm{g} / \mathrm{mL}$ PTX-CHORVG15-Lipo, PTX-CHO-Lipo or free PTX. After 24 $h$ incubation, the cells were washed with cold PBS, fixed with $4 \%$ paraformaldehyde for $20 \mathrm{~min}$, and stained with Hoechst 33258 at a concentration of $5 \mu \mathrm{g} / \mathrm{mL}$ for $15 \mathrm{~min}$. The apoptotic cells were observed and photographed under CLSM.

The apoptosis of C6 cells was quantitatively analyzed by Annexin V-FITC/PI double staining assay. The C6 cells were seeded in 6 -well plates $\left(15 \times 10^{4} /\right.$ well $)$, cultured for 24 $\mathrm{h}$, and then treated with free PTX, PTX-CHO-Lipo or PTX-CHO-RVG15-Lipo for additional 24 h. Subsequently, the cells were digested with $0.25 \%$ trypsin without EDTA, washed twice with cold PBS, centrifuged, resuspended in binding buffer, and treated per the protocol of Annexin V-fluorescein isothiocyanate (FITC)/ propidium iodide (PI) apoptosis detection kit. The percentage of apoptotic cells was analyzed by a FACSCalibur flow cytometer within $30 \mathrm{~min}$. 


\section{Wound-Healing Assay}

Wound-healing assay was conducted to assess the motility and migration ability of the tumor cells with treatment of different formulations. ${ }^{34}$ After proliferation for $24 \mathrm{~h}$ of $\mathrm{C} 6$ cells in 6-well plates, a wound field was produced in the middle of the well and the plates were washed with PBS to remove the floating cells. Then the cells were exposed to free PTX or PTX-CHO-RVG15-Lipo at a PTX concentration of $1 \mu \mathrm{g} / \mathrm{mL}$. Fresh medium was used as control. The healing status of scratch wound were observed and imaged at $0,12,24$, and $48 \mathrm{~h}$ using an inverted light microscope (Olympus, Hamburg, Germany).

\section{Glioma Model Establishment}

Male ICR mice (initially weighing 18-20 g) were commercially obtained from Vital River Laboratory Animal Technology Co., Ltd. (Beijing, P.R. China). All animal experiments were performed following the protocol approved by the Laboratory Animal Ethics Committee of the Institute of Materia Medica in CAMS and PUMC. The operational procedures abided by national and institutional principles and protocols for the care and use of experimental animals.

The orthotopic glioma bearing mouse model was developed according to a previously reported method. ${ }^{32}$ Briefly, C6 ${ }^{\text {luc }}$ cells (approximately $2 \times 10^{5}$ cells suspended in $4 \mu \mathrm{L}$ of PBS) were slowly implanted into the striatum $(1.8 \mathrm{~mm}$ right-lateral to the bregma and $3.0 \mathrm{~mm}$ in depth) of anesthetized ICR mice. Subsequently, the burr hole was sealed with bone wax and the scalp incision was sutured. The glioma-bearing mice were raised under standard condition for 1 week and used for following experiments.

\section{In vivo Imaging}

Glioma-bearing ICR mice were randomly divided into three groups $(n=3)$. DiR-Lip and DiR-RVG15-Lip was injected into the mice at a DiR dose of $10 \mu \mathrm{g} / \mathrm{kg}$ via tail vein. Normal saline was used as control. At 1, 4, 8 and 24 $\mathrm{h}$ post injection, the mice were anesthetized and then bioluminescence signals of the mice were acquired using an IVIS Spectrum CT in vivo imaging system (Caliper Life Sciences Inc., Mountain View, CA, USA). To further analyze the distribution of different formulations in the major organs, the main organs (liver, heart, spleen, lung and kidney) and tumor-bearing brains from the mice were separated for obtaining fluorescence images at 1, 8, 24 $\mathrm{h}$ after administration. The images were integrated using Living Image software (version 4.3.1; Caliper Life Sciences Inc.).

\section{Distribution of Targeted Liposomes in Glioma}

To explore the BBB penetration and the distribution in glioma of different liposomes, glioma-bearing ICR mice were intravenously injected with Cou-6-Lipo or Cou6-RVG15-Lipo at a dose of $1.5 \mathrm{mg}$ Cou- $6 / \mathrm{kg}$. At 3 $\mathrm{h}$ after injection, the mice were anesthetized and conducted heart perfusion with saline and $4 \%$ paraformaldehyde. ${ }^{19}$ Then the brains were removed and the glioma domains were frozen sectioned at a thickness of $10 \mathrm{~mm}$. Sections were stained with DAPI and washed with PBS. Finally, the sections were observed and imaged by CLSM.

\section{Anti-Glioma Efficacy}

The orthotopic glioma model was established as described in section 2.12 to perform tumor growth inhibition experiment. Seven days after tumor implantation, glioma-bearing ICR mice were randomly divided into four groups (six mice per group) and treated with free PTX, PTX-CHOLipo and PTX-CHO-RVG15-Lipo respectively at a PTX dose of $7.5 \mathrm{mg} / \mathrm{kg}$ via tail vein injection. Mice treated with saline were used as negative control. The treatments were repeated every 3 days for 5 times. Bioluminescence signals of the mice brain were monitored at 7, 13,16, 19 and $22 \mathrm{~d}$ post-implantation by an IVIS Spectrum CT in vivo imaging system. In addition, the body weight of mice was observed to assess the safety of different preparations. The mice were sacrificed by cervical dislocation at $22 \mathrm{~d}$ postimplantation. The brains were removed for terminaldeoxynucleotidyl transferase mediated nick end labeling (TUNEL) assays to assess tumor cell apoptosis. For evaluating the postoperative antimetastatic efficacy, the main organs (heart, liver, spleen, lungs, and kidneys) were collected and embedded in paraffin, sectioned, and then stained by hematoxylin and eosin (H\&E) for histopathological analysis.

The other mice (10 per group) were used to monitor survival times. Survival curves were plotted using GraphPad Prism software (version 5.0.0.0; GraphPad Software Inc., La Jolla, CA, USA). 

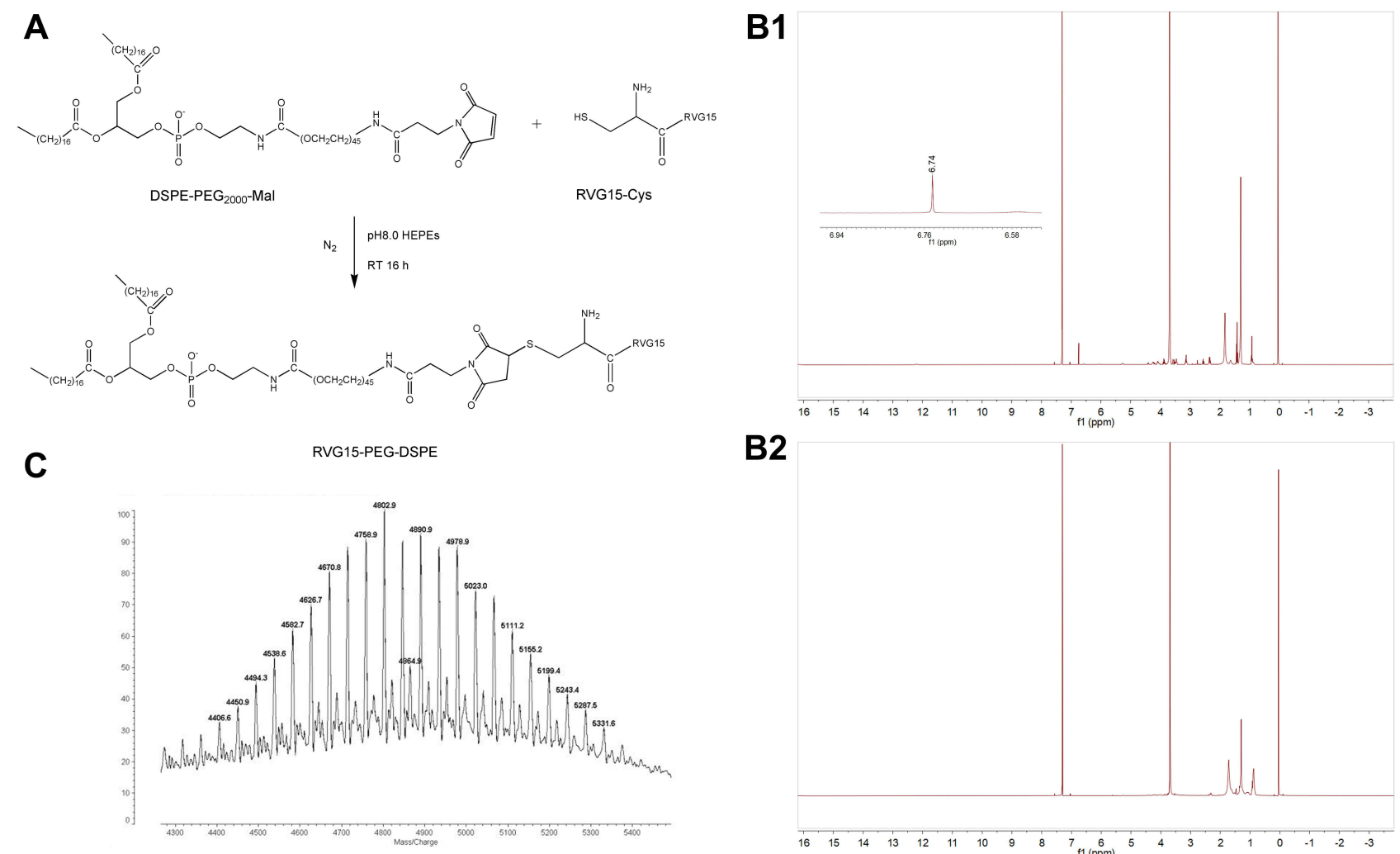

B2

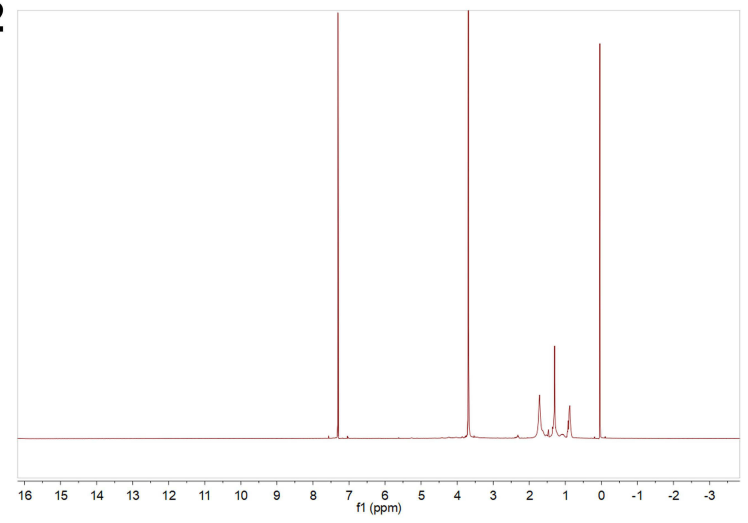

Figure 2 (A) Synthesis route of RVGI5-PEG-DSPE. (B) 'H NMR spectra of DSPE-PEG 2000 -Mal (B I); RVGI5-PEG-DSPE (B2). (C) MALDI-TOF-MS spectra of RVGI5-PEGDSPE.

Abbreviations: 'H NMR spectra, proton nuclear magnetic resonance spectroscopy; MALDI-TOF-MS spectra, matrix-assisted laser desorption/ionization time of flight mass spectrometry.

\section{Statistical Analysis}

The representative data are presented as mean \pm standard deviation (SD) calculated on Microsoft Excel. Statistical comparisons were analyzed to determine group differences through ANOVA by SPSS 24. Student's $t$-test was used to evaluate significant difference between two groups, indicated as follows: ${ }^{*} P<0.05,{ }^{* *} P<0.01,{ }^{* * *} P<0.001$.

\section{Results and Discussion}

\section{Synthesis and characterization of RVG I5- $\mathrm{PEG}_{2000^{-}}$DSPE}

The RVG15-PEG $2000-\mathrm{DSPE}$ were successfully prepared as illustrated in Figure 2A, in which the RVG15 peptide was conjugated to DSPE- $\mathrm{PEG}_{2000}$-Mal through an addition reaction. In detail, the maleimide on DSPE-PEG ${ }_{2000}-$ Mal reacted with the sulfhydryl group of the RVG15 peptide under the protection of nitrogen to obtain RVG15- $\mathrm{PEG}_{2000}$-DSPE. Figure $2 \mathrm{~B}$ shows the ${ }^{1} \mathrm{H}$ NMR spectra of DSPE-PEG ${ }_{2000^{-}}$ Mal and RVG15-PEG-DSPE in $\mathrm{CDCl}_{3}$ respectively.
Successful conjugation of RVG15-PEG-DSPE was verified by the disappearance of the double bond of maleimide at $6.74 \mathrm{ppm}$ compared to DSPE-PEG ${ }_{2000}$-Mal. The MALDITOF-MS spectrum (Figure 2C) showed the average MW of RVG15-PEG ${ }_{2000}$-DSPE was approximately $4800 \mathrm{Da}$, in line with its calculated MW, which indicated that the target molecule was successfully synthesized.

\section{Preparation of PTX-CHO Complex}

Given that PTX is extremely low solubility and poor stability alone in liposomes, PTX-CHO complex was prepared to improve entrapment efficiency and stability of PTX in the liposomes through the traditional solvent evaporation method. ${ }^{35}$ DSC analysis is a fast and reliable approach to readily analyze interactions between PTX and cholesterol in the PTX-CHO complex. The DSC thermograms of PTX, cholesterol, physical mixture of PTX and cholesterol and PTX-CHO complex were demonstrated in Figure 3. PTX (A) and cholesterol (B) exhibited endothermal peaks of $227.0^{\circ} \mathrm{C}$ and $148.2^{\circ} \mathrm{C}$, respectively. The physical mixture 


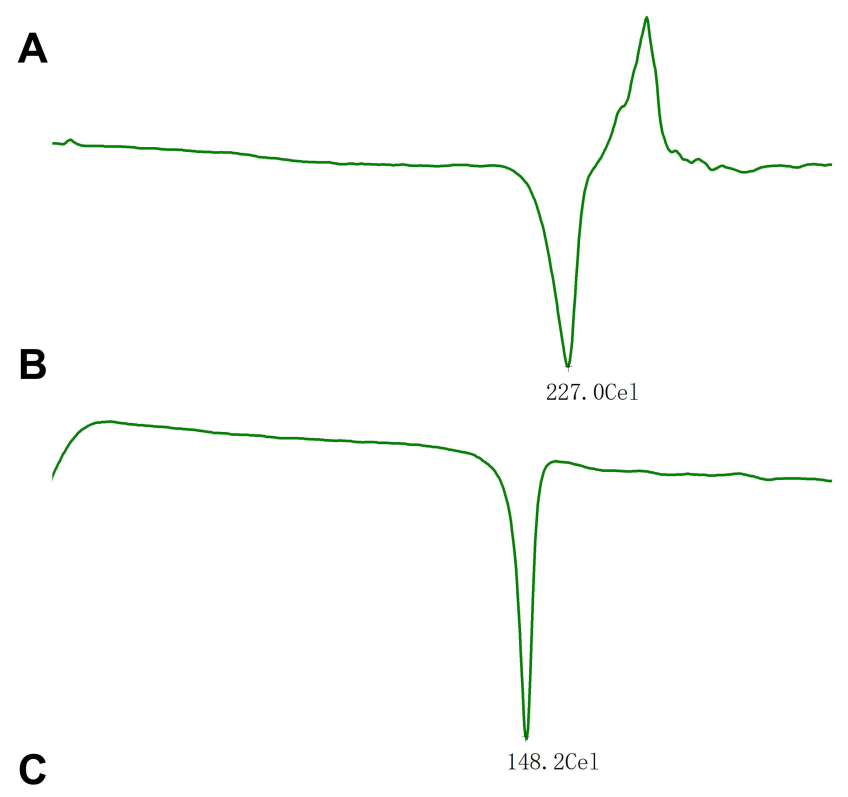

D

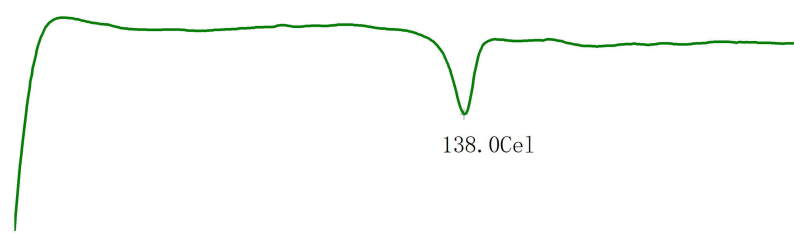

Figure 3 DSC curves of PTX (A), cholesterol (B), physical mixture of PTX and cholesterol (C), and PTX-CHO complex (D).

Abbreviations: DSC, differential scanning calorimetry; PTX, paclitaxel; PTX-CHO complex, paclitaxel-cholesterol complex.

of PTX and cholesterol (C) showed two separate endothermal peaks of PTX at $196.0^{\circ} \mathrm{C}$ and cholesterol at $149.2^{\circ} \mathrm{C}$. Compared with DSC thermograms of PTX and cholesterol, the endothermal peaks of PTX and cholesterol disappeared in the thermogram of PTX-CHO complex (D), replaced by a broad peak at $138.0^{\circ} \mathrm{C}$. The above results indicated the successful preparation of PTX-CHO complex.

\section{Characterization of Liposomes}

Proper sizes of nanoparticles were important for BBB penetrating and tumor targeting. ${ }^{36}$ DLS measurements demonstrated PTX-CHO encapsulated into RVG15-Lipo were able to readily form stable nanoparticles with the average particle size around $128.15 \pm 1.63 \mathrm{~nm}$ and PDI around $0.29 \pm 0.01$ (Figure 4A), demonstrating that the size distribution was homogeneous. PTX-CHO-RVG15-Lipo were negatively charged with zeta potential of $-15.55 \pm 0.78 \mathrm{mV}$ (Figure 4B). Studies had shown that NPs with surfaces containing a slightly negative charge might avoid nonspecific organ uptake and easily accumulate in tumor. The morphology of PTX-CHORVG15-Lipo was observed under TEM, and revealed that the desired nanoparticles were spherical structure with a smooth surface (Figure 4C). The minicolumn centrifugation technique was used to measure EE and DL, and the results showed that the $\mathrm{EE} \%$ and DL\% of PTX-CHO-RVG15-Lipo were $(98.76$ $\pm 0.08) \%$ and $(1.52 \pm 0.12) \%$, respectively.

\section{In vitro Paclitaxel Release Assay}

The in vitro release profiles of PTX-CHO-RVG15-Lipo and free PTX were characterized at $37{ }^{\circ} \mathrm{C}$ for $72 \mathrm{~h}$ in PBS ( $\mathrm{pH} 7.4$ ) containing $0.5 \%$ Tween 80. As shown in Figure 5, free PTX exhibited a relatively rapid and complete release, with nearly $80 \%$ of the drug released into the media within $48 \mathrm{~h}$ incubation. Meanwhile, PTX-CHORVG15-Lipo had sustained release behavior in the $72 \mathrm{~h}$, and the cumulative PTX release of drug loaded liposomes was only $40 \%$ after $48 \mathrm{~h}$ incubation in PBS.
A

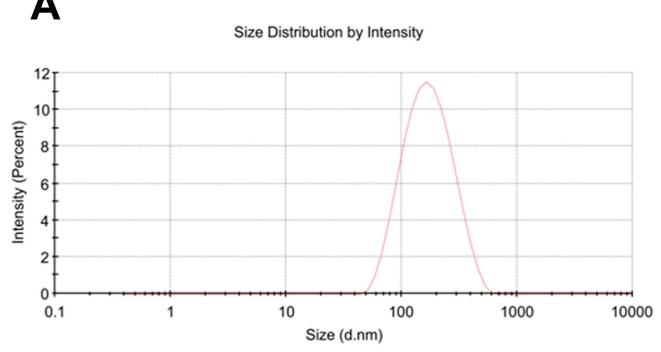

B

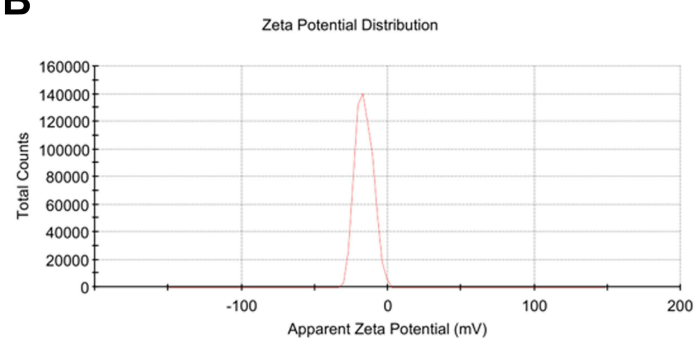

C

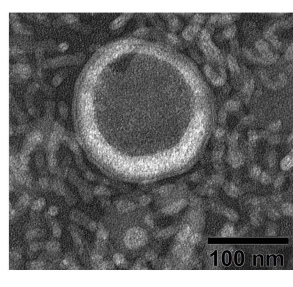

Figure 4 Characterizations of PTX-CHO-RVGI5-Lipo.

Notes: (A) Size distribution of PTX-CHO-RVGI5-Lipo. (B) Zeta potential distribution of PTX-CHO-RVGI5-Lipo. (C) TEM image of PTX-CHO-RVGI5-Lipo (scale bar: $100 \mathrm{~nm}$ ).

Abbreviations: PTX-CHO-RVGI5-Lipo, paclitaxel-cholesterol complex-loaded RVGI5-modified liposomes; TEM, transmission electron microscopy. 


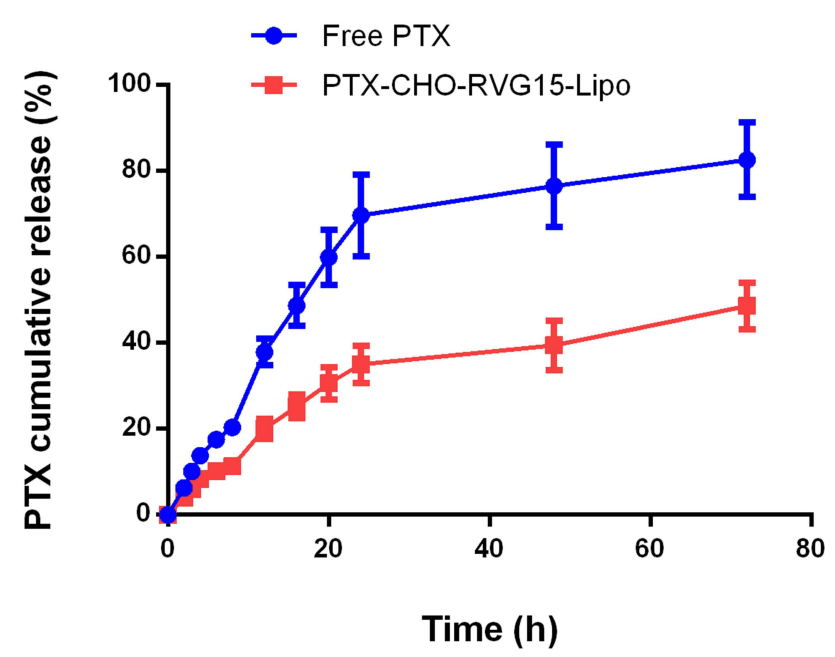

Figure 5 The PTX release profiles of free PTX and PTX-CHO-RVG I5-Lipo in PBS $(\mathrm{pH} 7.4)$ containing $0.5 \%$ Tween $80(n=3$, mean \pm SD).

Abbreviations: PTX, paclitaxel; PTX-CHO-RVGI5-Lipo, paclitaxel-cholesterol complex-loaded RVG15-modified liposomes.

\section{Cellular Uptake Studies Cellular Uptake on Different Cells}

The fluorescent probe coumarin-6 replaced PTX to prepare Cou-6-RVG15-Lipo and Cou-6-Lipo for evaluating the cellular uptake of different formulations in HBMEC and C6 cells which could respectively represent the $\mathrm{BBB}$ and glioma tumor. Then, the cellular uptake was investigated by both qualitative (CLSM) and quantitative (flow cytometry) methods. Figures $6 \mathrm{~A}$ and $7 \mathrm{~A}$ show the CLSM results in HBMEC cells and C6 cells, respectively. The blue and green fluorescence signals represented the DAPIstained cells nuclei and Cou-6, respectively. It was indicated that the green fluorescence signals could be evidently observed in both HBMEC and C6 cells after incubation with Cou-6-RVG15-Lipo for $15 \mathrm{~min}$ and the strength of signals at $15,60,120$, and $240 \mathrm{~min}$ had almost no changes. In comparison, the fluorescence signals of free Cou-6 and Cou-6-Lipo were weak which could not be clearly observed. Furthermore, at each time point, the uptake amount of RVG15-modified liposomes was greater than that of the unmodified groups into HBMEC and C6 cells.

Flow cytometry assay exhibited similar results with that obtained with CLSM. Cou-6-RVG15-Lipo showed the highest cellular uptake level on both HBMEC and C6 cells, with about 1.8-fold higher than Cou-6-Lipo and nearly 2.7-fold higher than free Cou-6 on HBMEC cells, and 1.7-fold and 3.4-fold on C6 cells (Figures 6B-E and
7B-E). RVG15-modified liposomes showed a significant improvement of cellular uptake, possibly attributing to the enhanced cell surface binding ability to nAChR of RVG15.

\section{Uptake Mechanism Study}

Possible cellular uptake mechanism of RVG15-modified liposomes was explored in C6 glioma cells via different endocytosis inhibitors, including clathrin-mediated endocytosis inhibitor (chlorpromazine hydrochloride, CPZ), microtubule protein inhibitor (colchicine, $\mathrm{Col}$ ), and caveolaemediated endocytosis inhibitor (methyl-beta-cyclodextrin, M- $\beta$-CD). ${ }^{37}$ As depicted in Figure $7 F$, cellular uptake of Cou-6-RVG15-Lipo was significantly suppressed by CPZ with the relative reduced fluorescence intensity of about $58.55 \%$, indicating that the endocytosis of RVG15modified liposomes was more mediated by grid proteins. Compared with the control group, the cellular uptake of Cou-6-RVG15-Lipo after treatment with Col and M- $\beta-C D$ barely decreased with fluorescence intensity of $83 \%$ and $73 \%$ respectively, suggesting that both microtubule protein and lipid rafts might not involve in the cellular uptake of RVG15-modified liposomes. Collectively, these results suggested that Cou-6-RVG15-Lipo entered cells via the endocytic pathways of clathrin-mediated endocytosis.

\section{Cell Viability Assay}

The cytotoxicity of blank liposomes was detected by CCK8 assay. The blank liposomes exhibited no significant cytotoxicity in both HBMEC cells (Figure 8A) and C6 cells (Figure $8 \mathrm{~B}$ ) with viabilities all higher than $85 \%$ after $24 \mathrm{~h}$ or $48 \mathrm{~h}$ incubation, suggesting that blank liposomes were safe and biocompatible for further use in vivo. Subsequently, CCK-8 assay was also used to assess the inhibitory capacity of the various PTX formulations against C6 cell proliferation after incubation for $24 \mathrm{~h}$ or $48 \mathrm{~h}$. As exhibited in Figure 8C, free PTX, PTX-CHO-Lipo and PTX-CHORVG15-Lipo exhibited dose-dependent inhibition for C6 cell proliferation. PTX-CHO-RVG15-Lipo could inhibit cell proliferation more effectively at concentrations of PTX ranging from $0.01 \mu \mathrm{g} / \mathrm{mL}$ to $50 \mu \mathrm{g} / \mathrm{mL}$ after 24 or $48 \mathrm{~h}$ incubation compared with free PTX and PTX-CHOLipo. Furthermore, the $\mathrm{IC}_{50}$ value for free PTX, PTX-CHOLipo and PTX-CHO-RVG15-Lipo were $8.009 \mu \mathrm{g} / \mathrm{mL}$, $4.587 \mu \mathrm{g} / \mathrm{mL}$ and $0.091 \mu \mathrm{g} / \mathrm{mL}$ after $24 \mathrm{~h}$ incubation, respectively. The $\mathrm{IC}_{50}$ of PTX-CHO-RVG15-Lipo was markedly lower than free PTX and PTX-CHO-Lipo. The significantly enhanced anti-proliferative effect of PTX-CHO -RVG15-Lipo was related to higher cellular internalization 
A

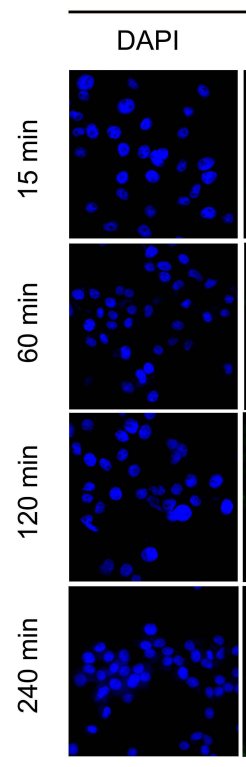

B

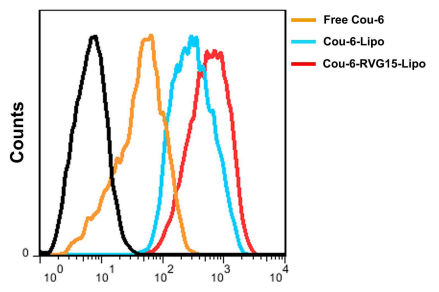

Free Cou-6

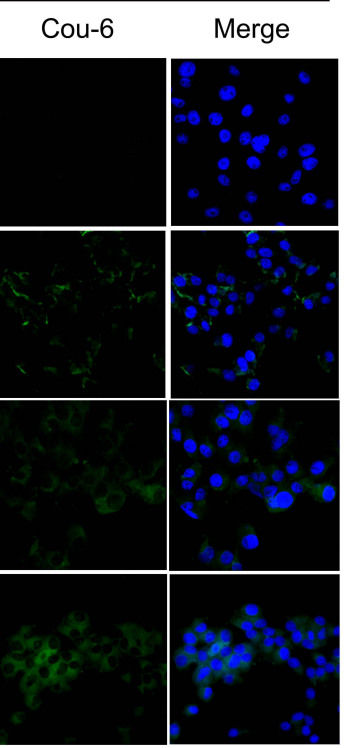

C

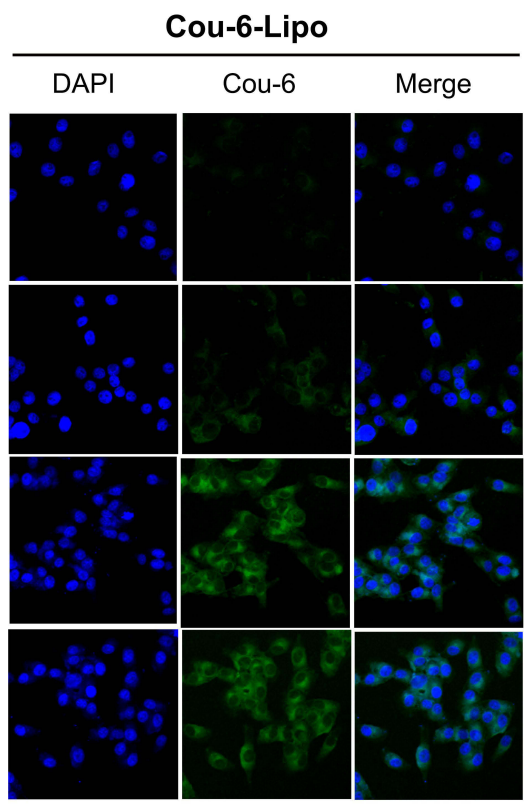

D

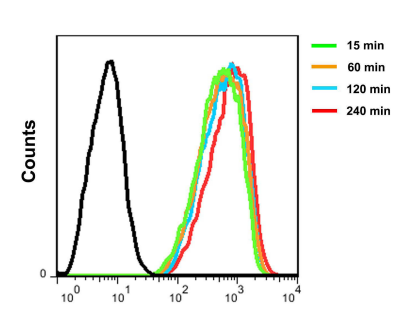

Cou-6-RVG15-Lipo

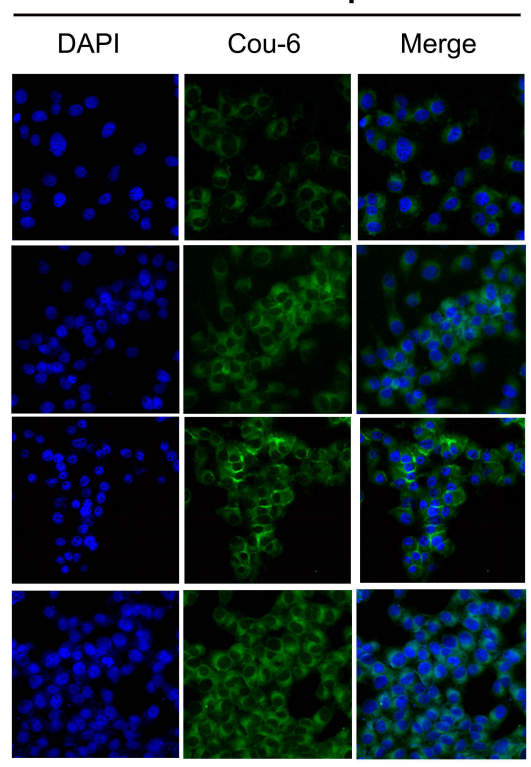

E

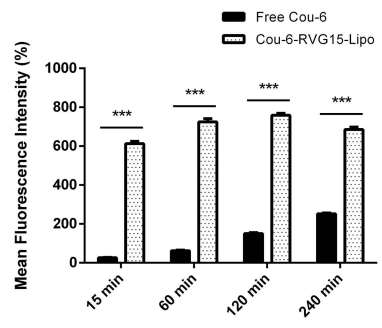

Figure 6 Cellular uptake of liposomes on HBMEC cells.

Notes: (A) Confocal images of cellular uptake after treatment with free Cou-6, Cou-6-Lipo or Cou-6-RVGI5-Lipo for 15, 60, I20 and 240 min, respectively. Analysis of cellular uptake of the control, free Cou-6, Cou-6-Lipo and Cou-6-RVGI5-Lipo by flow cytometry (B) and mean fluorescence intensity (C). Analysis of cellular uptake of Cou-6-RVG I5-Lipo incubation for 15, 60, 120 and 240 min by flow cytometry (D) and mean fluorescence intensity (E). Means \pm SD, $n=3$; ***P < 0.00I. Green: coumarin-6, blue: DAPI (nuclei).

Abbreviations: Cou-6, coumarin-6; Cou-6-RVGI5-Lipo, coumarin-6-loaded RVG I5-modified liposomes.

of RVG15-modified liposomes than free PTX and unmodified liposomes, as demonstrated in cellular uptake evaluation. Taking together, the above results suggested that PTX-CHO-RVG15-Lipo displayed stronger cytotoxicity than free PTX and PTX-CHO-Lipo, which may have better curative effect on tumor in vivo.

\section{Drug Penetration in the BBB Model}

With TEER of the tight monolayer above $200 \Omega \cdot \mathrm{cm}^{2}$, in vitro BBB model was successfully established to measure the transport efficiency of the different PTX formulations. Figure 9 shows that the transport efficiency increased with the prolongation of incubation time. After 180 min incubation, the cumulative transport efficiency was $16.82 \%, 8.31 \%$ and $13.12 \%$ for PTX-CHO-RVG15Lipo, free PTX and PTX-CHO-Lipo, respectively. PTXCHO-RVG15-Lipo showed the highest transport ratio, which was 2.0 and 1.3-fold higher than that for free PTX and PTX-CHO-Lipo, demonstrating that RVG15-modified liposomes exerted a significant ability to penetrate the BBB. The high BBB permeation of RVG15-modified liposomes might probably be attributed to high cellular uptake in HBMEC cells via nAChR-mediated transcytosis.

\section{Cell Apoptosis Study in vitro}

Hoechst staining assay showed that nuclear morphology changed including nuclear fragmentation, chromatin condensation and chromosome abnormality after treatment 
A

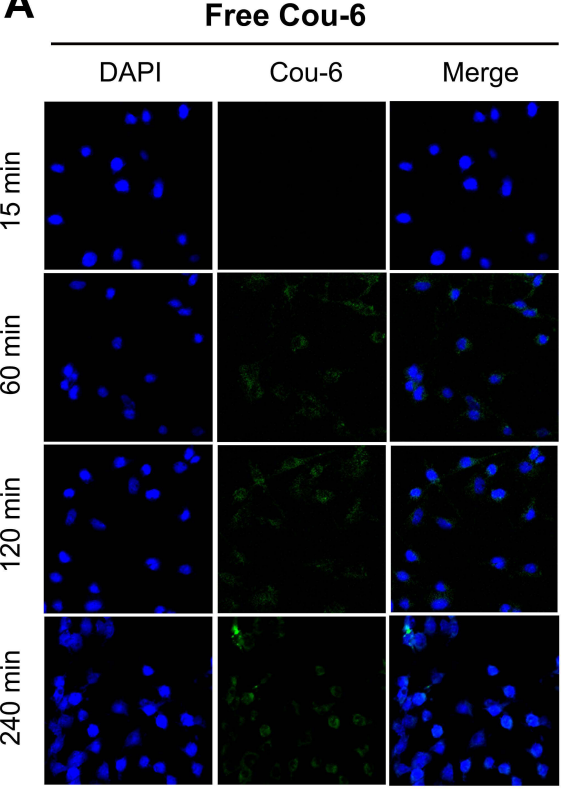

Cou-6-Lipo
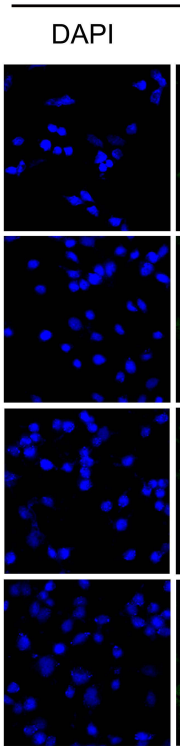

C
B

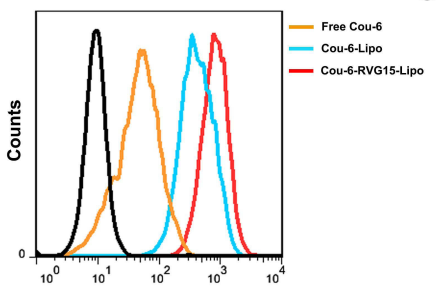

D

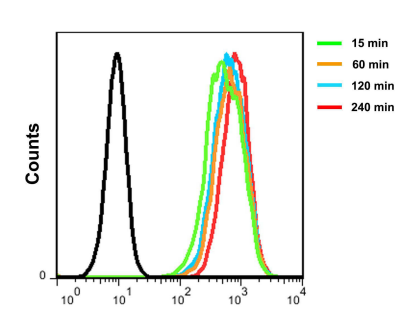

E

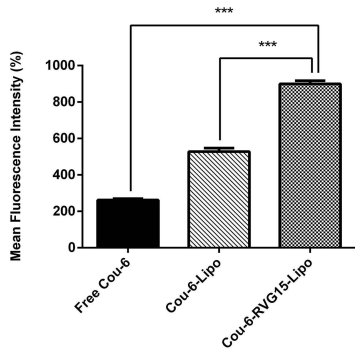

- Free Cou-6 Cou-6-RVG15-Lipo

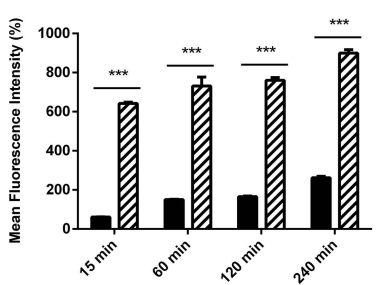

\section{Cou-6-RVG15-Lipo}

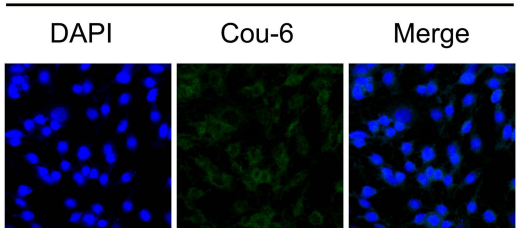

50

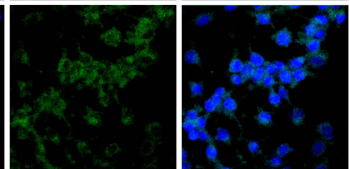

50
89
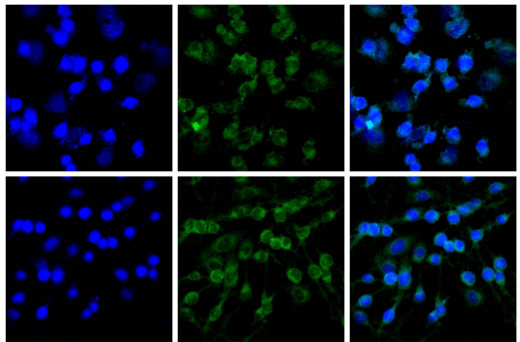

Figure 7 Cellular uptake of liposomes on C6 cells.

Notes: (A) Confocal images of cellular uptake after treatment with free Cou-6, Cou-6-Lipo or Cou-6-RVGI5-Lipo for 15, 60, I20 and 240 min, respectively. Analysis of cellular uptake of the control, free Cou-6, Cou-6-Lipo and Cou-6-RVGI5-Lipo by flow cytometry (B) and mean fluorescence intensity (C). Analysis of cellular uptake of Cou-6-RVG I5-Lipo incubation for 15, 60, 120 and $240 \mathrm{~min}$ by flow cytometry (D) and mean fluorescence intensity (E). (F) Cellular uptake analysis of Cou-6-RVGI5-Lipo after incubation with different endocytic inhibitors by flow cytometry. Means \pm SD, $n=3$; ***P < 0.00I. Green: coumarin-6, blue: DAPI (nuclei).

Abbreviations: Cou-6, coumarin-6; Cou-6-RVGI5-Lipo, coumarin-6-loaded RVGI5-modified liposomes; CPZ, chlorpromazine; Col, colchicine; M- $\beta$-CD, methyl- $\beta$ cyclodextrin.

with free PTX, PTX-CHO-Lipo and PTX-CHO-RVG15Lipo (Figure 10A). The images displayed that the amount of perinuclear apoptotic bodies and multinucleated cells in PTX-CHO-RVG15-Lipo treated group was more than that with free PTX and PTX-CHO-Lipo.

The quantification of the cell apoptosis was further detected using the Annexin-V/PI double staining method by flow cytometry. As shown in $10 \mathrm{~B}$ and $\mathrm{C}$, the cell apoptosis percentage of PTX-CHO-RVG15Lipo group was $(34.70 \% \pm 0.43 \%)$, significantly higher than that of free PTX group $(14.88 \% \pm 0.80 \%)$ $(* * * P<0.001)$ and PTX-CHO-Lipo group $(18.57 \% \pm$ $3.95 \%)(* * P<0.01)$. Generally, the significant cell apoptosis of PTX-CHO-RVG15-Lipo arose from the effective cellular uptake and sequentially enhanced anti-proliferative effect. 
A

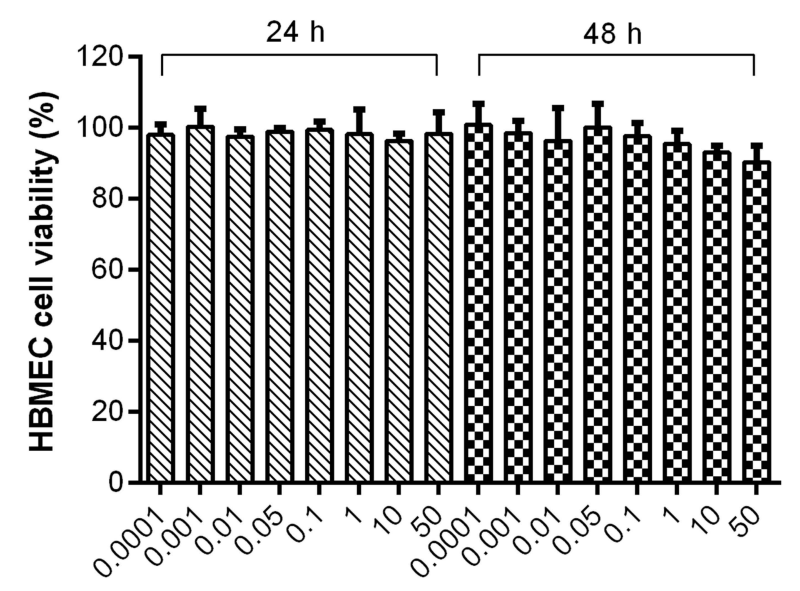

Equivalent concentration to

PTX-CHO-RVG15-Lipo $(\mu \mathrm{g} / \mathrm{ml})$
B

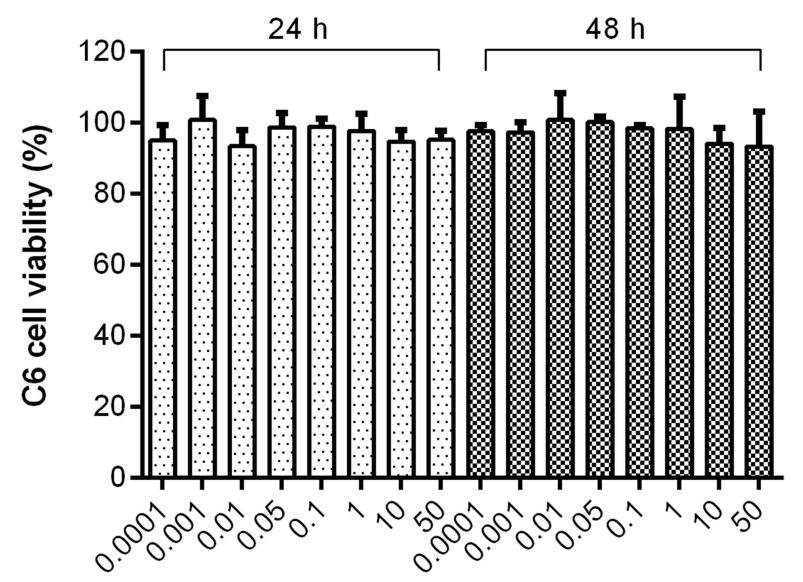

Equivalent concentration to PTX-CHO-RVG15-Lipo $(\mu \mathrm{g} / \mathrm{ml})$

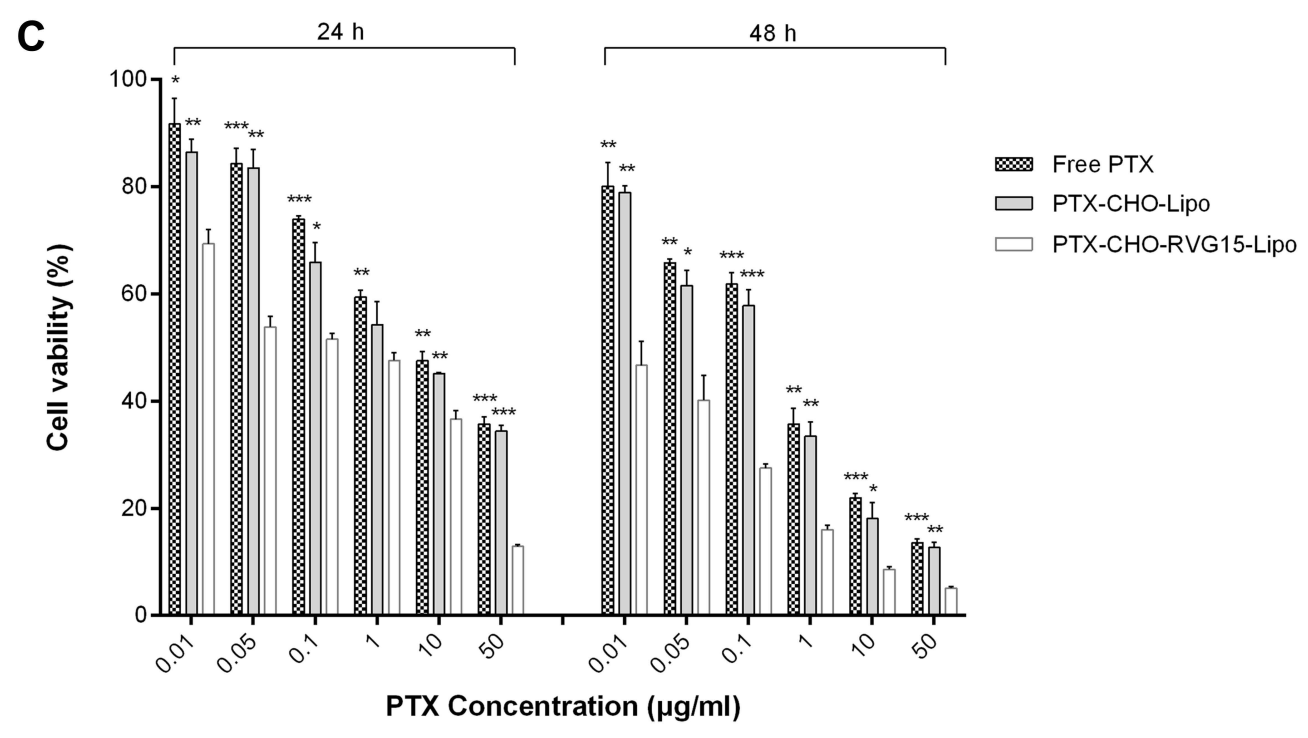

Figure 8 In vitro cytotoxicity of blank liposome at equivalent concentration to PTX-CHO-RVG 5 -Lipo ranging from $0.000 \mathrm{I} \mu \mathrm{g} / \mathrm{mL}$ to $50 \mu \mathrm{g} / \mathrm{mL}$ for 24 or $48 \mathrm{~h}$ on HBMEC cells $(\mathbf{A})$ or $\mathrm{C} 6$ cells $(\mathbf{B})(\mathrm{n}=3$, Means \pm SD). (C) Inhibitory capacity of PTX-CHO-RVG I5-Lipo, PTX-CHO-Lipo and free PTX against C6 cell proliferation after incubation of 24 or 48 h. Means \pm SD, $\mathrm{n}=3$; $* P<0.05$, $* * P<0.01$, *** $P<0.001$ versus the PTX-CHO-RVGI5-Lipo group.

Abbreviations: PTX, paclitaxel; PTX-CHO-Lipo, paclitaxel-cholesterol complex-loaded liposomes; PTX-CHO-RVGI5-Lipo, paclitaxel-cholesterol complex-loaded RVGI5modified liposomes.

\section{Wound-Healing Assay}

Wound-healing assay was carried out to intuitively evaluate random cell migration and invasion. As shown in Figure 11, the scratches in the control group gradually healed. In contrast, the cell density near the scratch decreased followed by a large number of apoptotic or dead cells, the cell morphology became irregular, and the scratches remained clearly visible after treatment with free PTX, PTX-CHO-Lipo and PTX-CHO-RVG15-Lipo. The results indicated that PTX significantly inhibited C6 cell migration, thus distinctly reducing the degree of wound healing. Importantly, PTX-CHO-RVG15-Lipo exhibited stronger anti-migration effect than free PTX and PTXCHO-Lipo. 


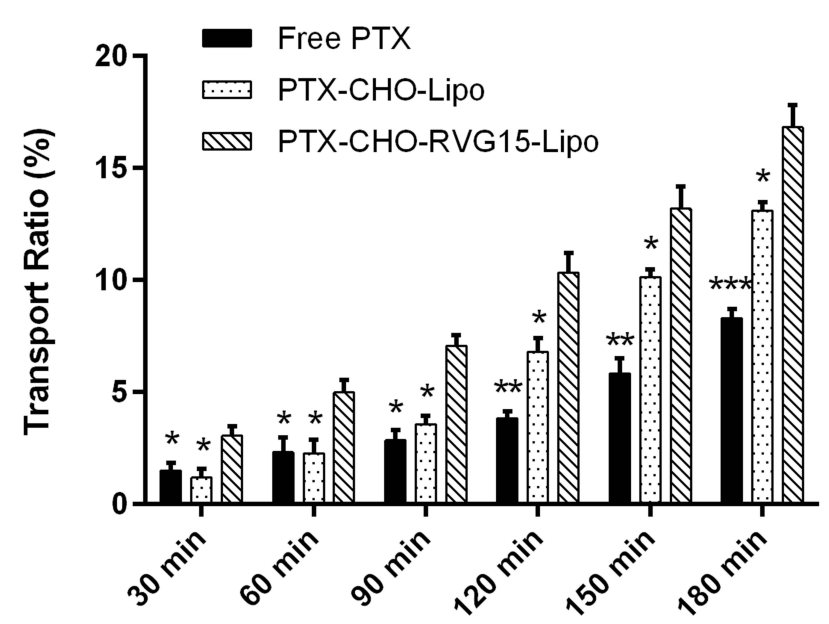

Figure 9 The transport ratios of PTX across the BBB model at predetermined times. Means $\pm S D, \mathrm{n}=3 ; * P<0.05$, $* * P<0.01$ and $* * * P<0.001$ versus the PTXCHO-RVG I5-Lipo group.

Abbreviations: PTX, paclitaxel; PTX-CHO-Lipo, paclitaxel-cholesterol complexloaded liposomes; PTX-CHO-RVGI5-Lipo, paclitaxel-cholesterol complex-loaded RVGI5-modified liposomes.

\section{Biodistribution in vivo}

The orthotopic glioma mouse model was developed to evaluate the BBB penetration ability and the glioma targeting efficiency of different liposomes in vivo. Figure 12A and B depicted the real-time in vivo images and the corresponding quantitative fluorescence results of glioma-bearing mice at different time points after DiR-loaded liposome injection. It was perspicuously observed that DiR-RVG15-Lipo group exhibited stronger fluorescent signals than that of the DiR-Lipo group in glioma site at any time point after systemic administration, consistent with the quantitative results. Moreover, Figure 12A shows DiR-RVG15-Lipo and DiR-Lipo both achieved maximum deposition in the brains at $1 \mathrm{~h}$ post injection, and the strong fluorescence signals of DiR-RVG15-Lipo in the brains could maintain for $24 \mathrm{~h}$ while the signals of DiR-Lipo obviously weakened. The mice were sacrificed at 1, 8, 24 $\mathrm{h}$ after injection, then the brains, hearts, livers, spleens, lungs and kidneys were harvested and pictured. As illustrated in Figure $12 \mathrm{C}$, the brain fluorescence intensity of the mice in the DiR-RVG15-Lipo group was obviously higher than that in the DiR-Lipo group. Both DiR-Lipo and DiR-RVG15-Lipo were mainly distributed in macrophage-related organs, such as the liver and the spleen, and presented less in other organs. ${ }^{38-41}$

Collectively, these results indicated that RVG15modified liposomes enhanced the BBB penetration efficiency and prolonged the residence time of the drug in brain after intravenous injection, and the same outcomes were observed in the prior cellular uptake assay (Figures 6 and 7) and BBB permeability study (Figure 9). Moreover, the presence of PEG in the structure prolonged the circulation time of liposomes in vivo, and the fluorescence intensity did not rapidly decline within $24 \mathrm{~h}$, thereby improving the efficacy of permeability and delivery.

\section{Distribution of Targeted Liposomes in Glioma}

To further illustrate the targeting effect of RVG15modified liposomes in vivo, we compared biological distribution in tumor tissues of glioma-bearing mice treated with Cou-6-Lipo and Cou-6-RVG15-Lipo, with Cou-6 as a tracer. The brain tissue was harvested after $3 \mathrm{~h}$ treatment, and then frozen sections were prepared to observe the distribution of liposomes in tumor tissue by confocal laser scanning microscopy. As shown in Figure 13, Cou-6-Lipo group had little distribution in glioma region, probably due to the poor cellular uptake and the low BBB permeation. By contrast, the Cou6-RVG15-Lipo group showed better BBB penetration and higher distribution in the glioma as a result of nAChR-mediated transcytosis. These results confirmed that RVG15-modified liposomes could significantly cross the BBB, accumulate within the tumor and penetrate deeply in tumor parenchyma.

\section{In vivo Anti-Glioma Efficacy}

The anti-glioma activities of different PTX formulations were evaluated on orthotopic $\mathrm{C}^{\text {luc }}$ glioma-bearing mice. At $7,10,13,16,19$ and $22 \mathrm{~d}$ post-implantation, the mice were injected with saline, free PTX, PTX-CHOLipo and PTX-CHO-RVG15-Lipo via tail vein, respectively. Meanwhile, an IVIS Spectrum CT in vivo imaging system was used to monitor glioma growth through bioluminescence signals of the mice brain. As shown in Figure 14A, PTX-CHO-RVG15Lipo had the strongest glioma inhibition effect. By contrast, the anti-glioma effect of free PTX and PTX-CHO-Lipo was evidently weak. The quantitative fluorescence results were shown in Figure 14B, which further confirmed the above tendency. At the third day after the final treatment, the mice were sacrificed to remove the brain tissues, and then the brain tissues were observed and photographed to evaluate the anti- 
A

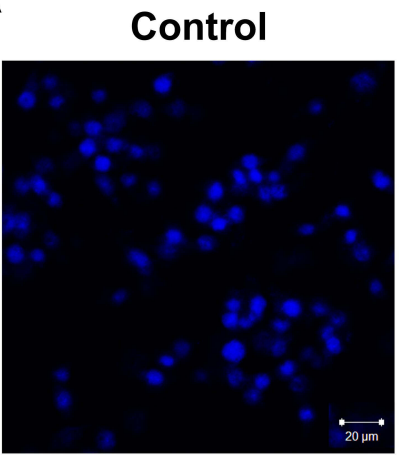

Free PTX

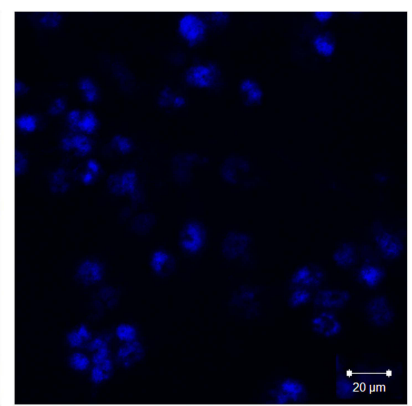

PTX-CHO-Lipo

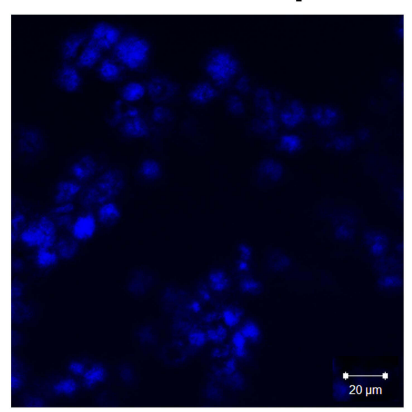

\section{$A^{\mathrm{B} 1}$}

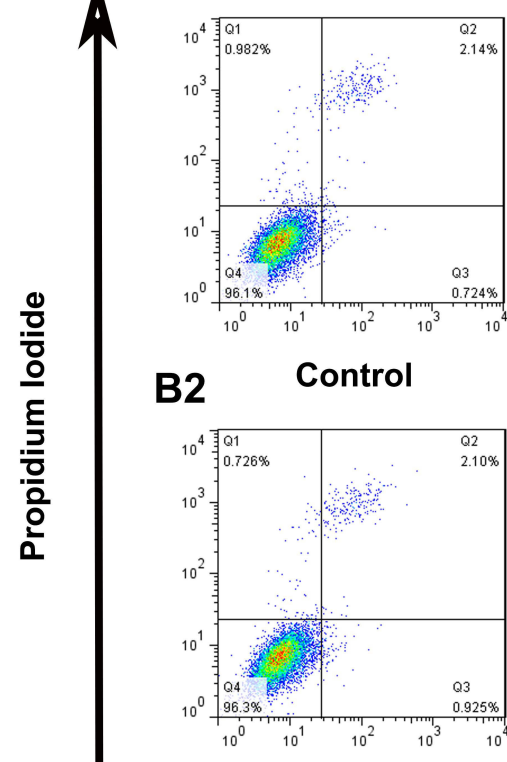

Blank liposome
C

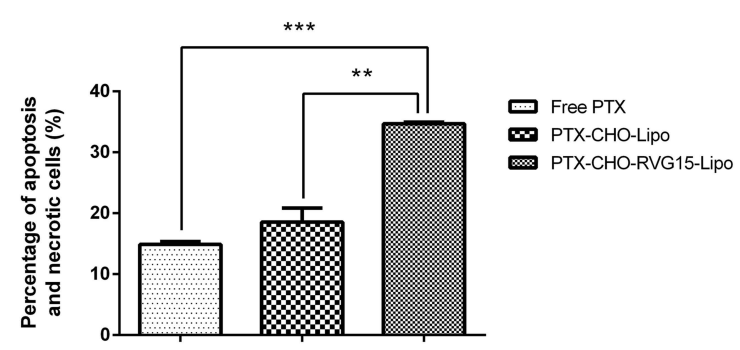

B4
PTX-CHO-RVG15-Lipo

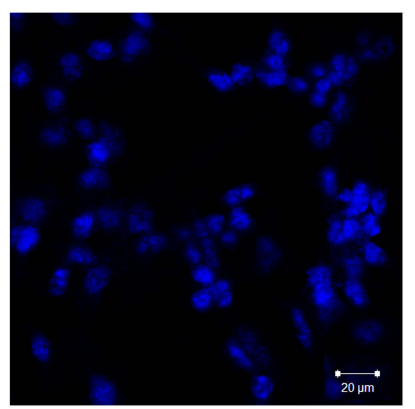

B3

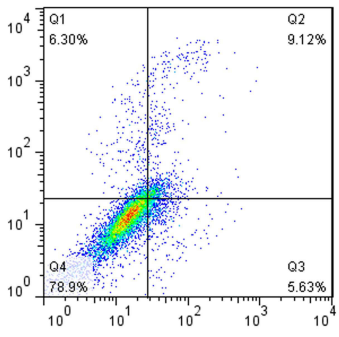

Free PTX

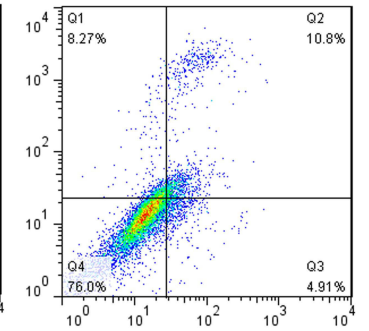

PTX-CHO-Lipo
B5

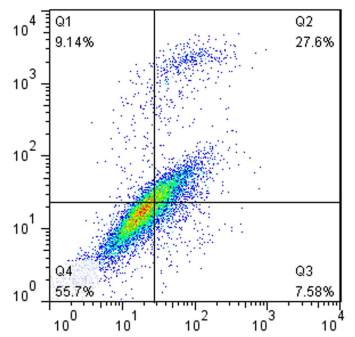

PTX-CHO-RVG15-Lipo

\section{Annexin V-FITC}

Figure 10 Cell apoptosis on C6 cells.

Notes: (A) Hoechst staining (magnification $\times 200$ ). Scale bar: $20 \mu \mathrm{m}$. (B) The apoptosis study of $\mathrm{C} 6$ cells after $24 \mathrm{~h}$ incubation with control (B I); blank liposome (B2); free PTX (B3); PTX-CHO-Lipo (B4) and PTX-CHO-RVGI5-Lipo (B5). (C) The percentage of apoptosis after free PTX, PTX-CHO-Lipo and PTX-CHO-RVGI5-Lipo treatment $(\mathrm{n}=3$, Means $\pm \mathrm{SD}), * * P<0.01$, ***P $<0.001$.

Abbreviations: PTX, paclitaxel; PTX-CHO-Lipo, paclitaxel-cholesterol complex-loaded liposomes; PTX-CHO-RVGI5-Lipo, paclitaxel-cholesterol complex-loaded RVG I5modified liposomes.

glioma effect (Figure 14C). In consistent with the previous results, the anti-glioma effect of the PTX-CHO -RVG15-Lipo was evidently superior than that of free PTX and PTX-CHO-Lipo with the relatively small tumor volume, less hemorrhaging and necrosis (red circle). Therefore, PTX-CHO-RVG15-Lipo exhibited significantly potent glioma-suppressing activity probably due to stronger BBB permeation, deeper glioma penetration and higher cellular uptake.
The body weight of the mice was measured every 3 days to evaluate the progress of glioma proliferation and the systemic toxicity of different formulations. No noticeable body weight change of mice was observed during treatment with PTX-CHO-RVG15-Lipo, free PTX and PTX-CHO-Lipo treated groups showed apparent body weight loss (Figure 14D). The results indicated that PTX-CHO-RVG15-Lipo could suppress the rapid growth of the glioma more effectively with little 


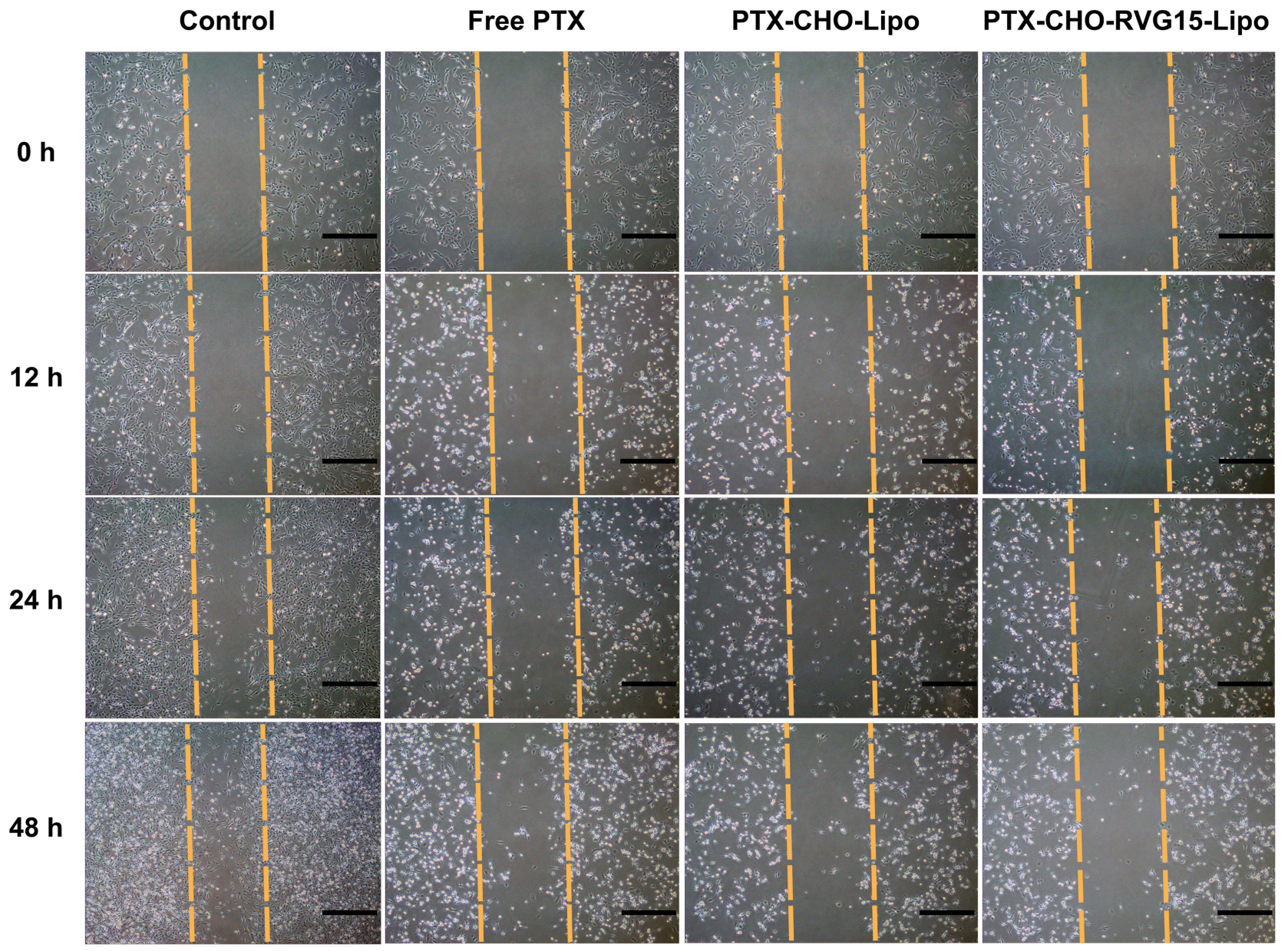

Figure II Wound-healing assay on C6 cells; scale bar $=100 \mu \mathrm{m}$.

Abbreviations: PTX, paclitaxel; PTX-CHO-Lipo, paclitaxel-cholesterol complex-loaded liposomes; PTX-CHO-RVG I5-Lipo, paclitaxel-cholesterol complex-loaded RVG I5modified liposomes.

side effect for glioma-bearing mice at the tested dose level.

Figure 15A shows the results of TUNEL assay that the PTX-CHO-RVG15-Lipo group revealed extensive necrosis and apoptosis in the tumor of mice with large quantity of brown TUNEL-positive cells, suggesting that PTXCHO-RVG15-Lipo could penetrate deeply into the glioma tissues and kill tumor cells. By comparison, the rate of apoptosis cells was lower in the PTX-CHO-Lipo group. What's more, the free PTX group showed much less drug activity in the tumor site owing to poor solubility and incapable of reaching the interior of tumor tissue. Hence, PTX-CHO-RVG15-Lipo exhibited the best anti-glioma activity among all groups.

The orthotopic glioma model spontaneously formed lung and liver metastases, indicating the progression of tumor metastasis. The antimetastatic capacity of PTXCHO-RVG15-Lipo was evaluated by histological analysis by H\&E staining. The major organs, including heart, liver, spleen, lung and kidney, were removed to prepare paraffin-embedded sections after treatment. The pathological images of lung and liver tissues are presented in Figure 15B. Vascular infiltration was found obviously in the saline, free PTX and PTX-CHO-Lipo groups (black arrow), whereas no abnormalities were observed in the PTX-CHO-RVG15-Lipo group. Unlike the saline, free PTX and PTX-CHO-Lipo groups in which tumor metastases were observed in the liver (black dotted circle), the PTX-CHO-RVG15-Lipo group showed no distinguishable tumor metastasis in the liver tissue. The results indicated that PTX-CHO-RVG15-Lipo exhibited a significant ability to suppress tumor metastasis. As shown in Figure 15C, 

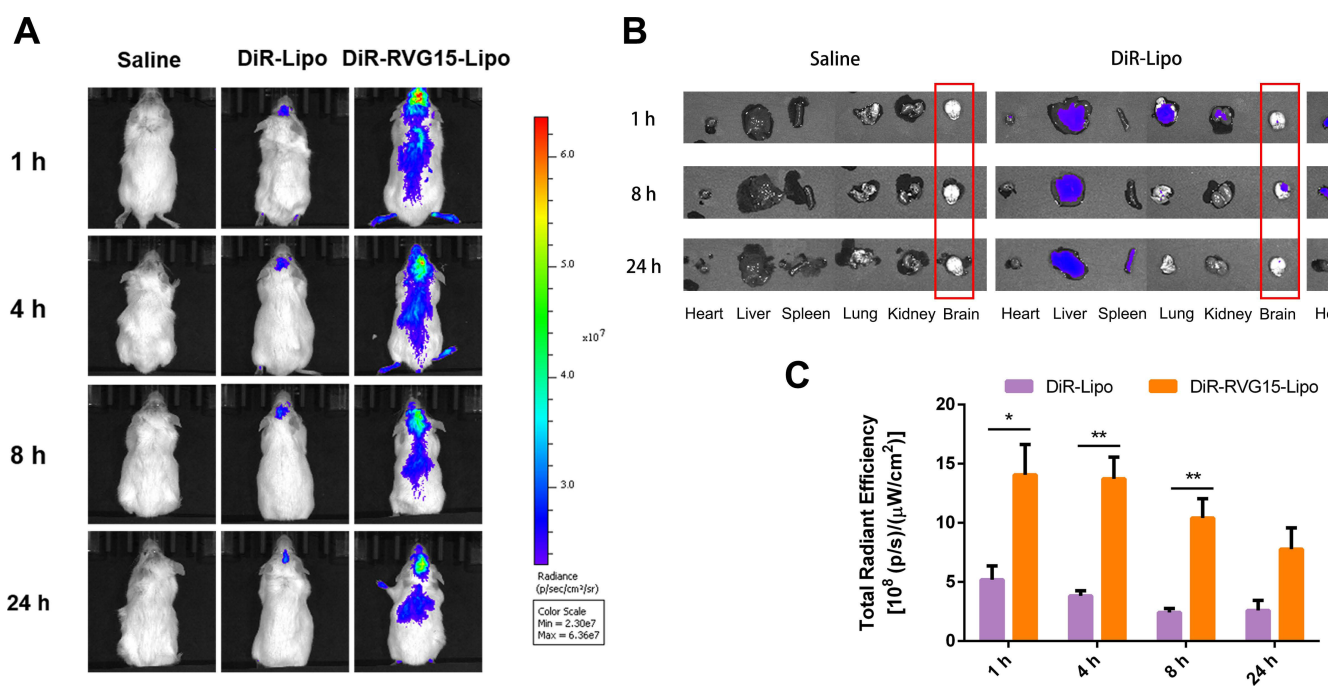

Figure 12 In vivo distribution.

Notes: (A) In vivo fluorescence imaging of C6 ${ }^{\text {luc }}$ orthotopic glioma-bearing mice treated with saline, DiR-Lipo and DiR-RVG I5-Lipo at I h, 4 h, 8 h and 24 h. (B) Images of the brain and other organs at I h, $8 \mathrm{~h}$ and $24 \mathrm{~h}$. (C) In vivo radiant efficiency of C6 ${ }^{\text {luc }}$ orthotopic glioma-bearing mice at I h, $4 \mathrm{~h}, 8 \mathrm{~h}$ and $24 \mathrm{~h}$. Means $\pm \mathrm{SD}, \mathrm{n}=3 ; * \mathrm{P}<0.05$, $* * P<0.01$.

Abbreviations: DiR-Lipo, DiR-loaded liposomes; DiR-RVGI5-Lipo, DiR-loaded RVGI5-modified liposomes.

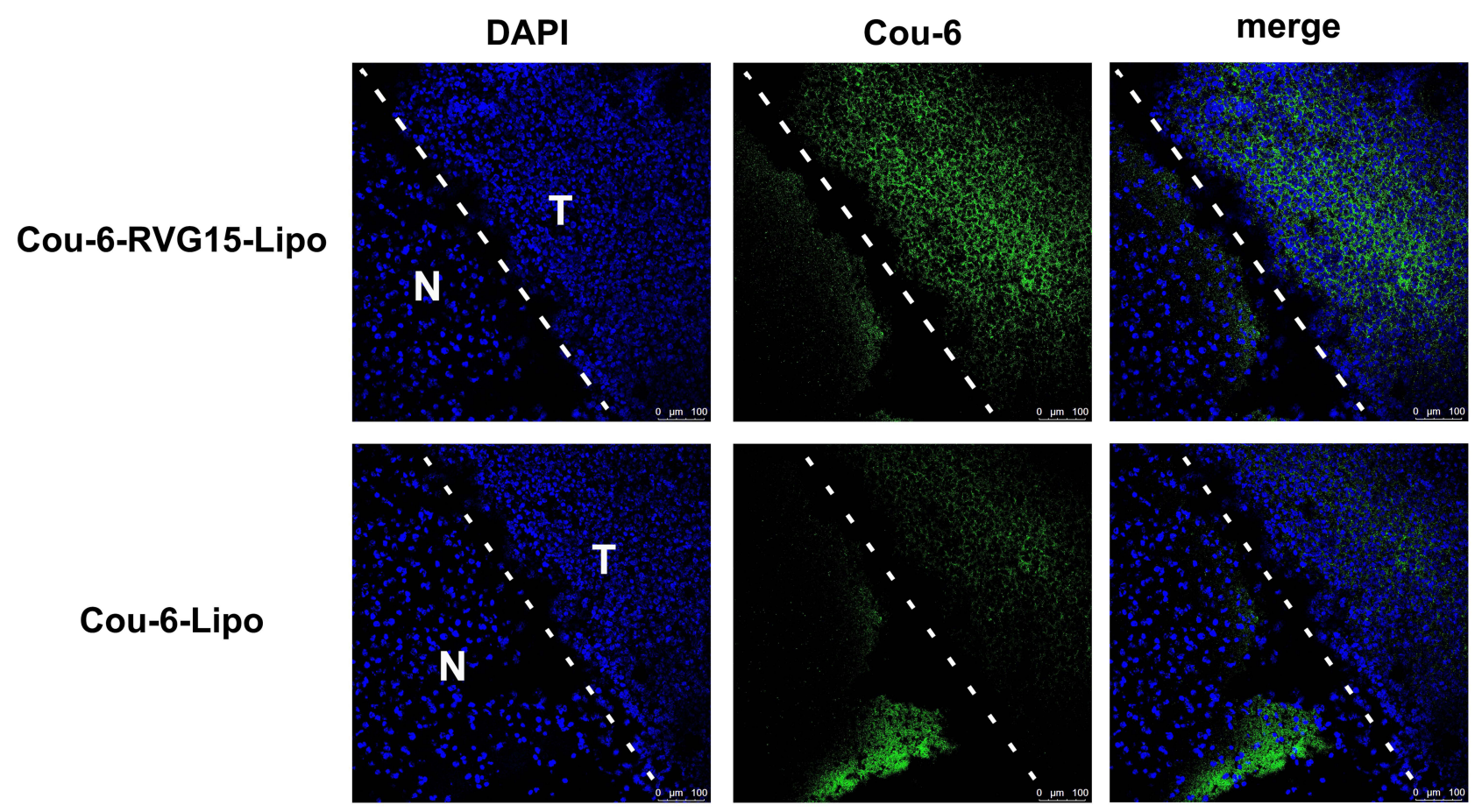

Figure 13 Tumor penetration behavior of Cou-6-Lipo and Cou-6-RVGI5-Lipo by CLSM. Tumor sections from C6 ${ }^{\text {luc }}$ orthotopic glioma-bearing mice following 3 h tail vein injection of Cou-6-Lipo and Cou-6-RVGI5-Lipo. Green: coumarin-6, blue: DAPI (nuclei). Scale bar: $100 \mu \mathrm{m}$.

Abbreviations: Cou-6, coumarin-6; Cou-6-Lipo, coumarin-6-loaded liposomes; Cou-6-RVGI5-Lipo, coumarin-6-loaded RVGI5-modified liposomes; CLSM, confocal laser scanning microscope; $\mathrm{N}$, normal brain tissue; $\mathrm{T}$, tumor. 


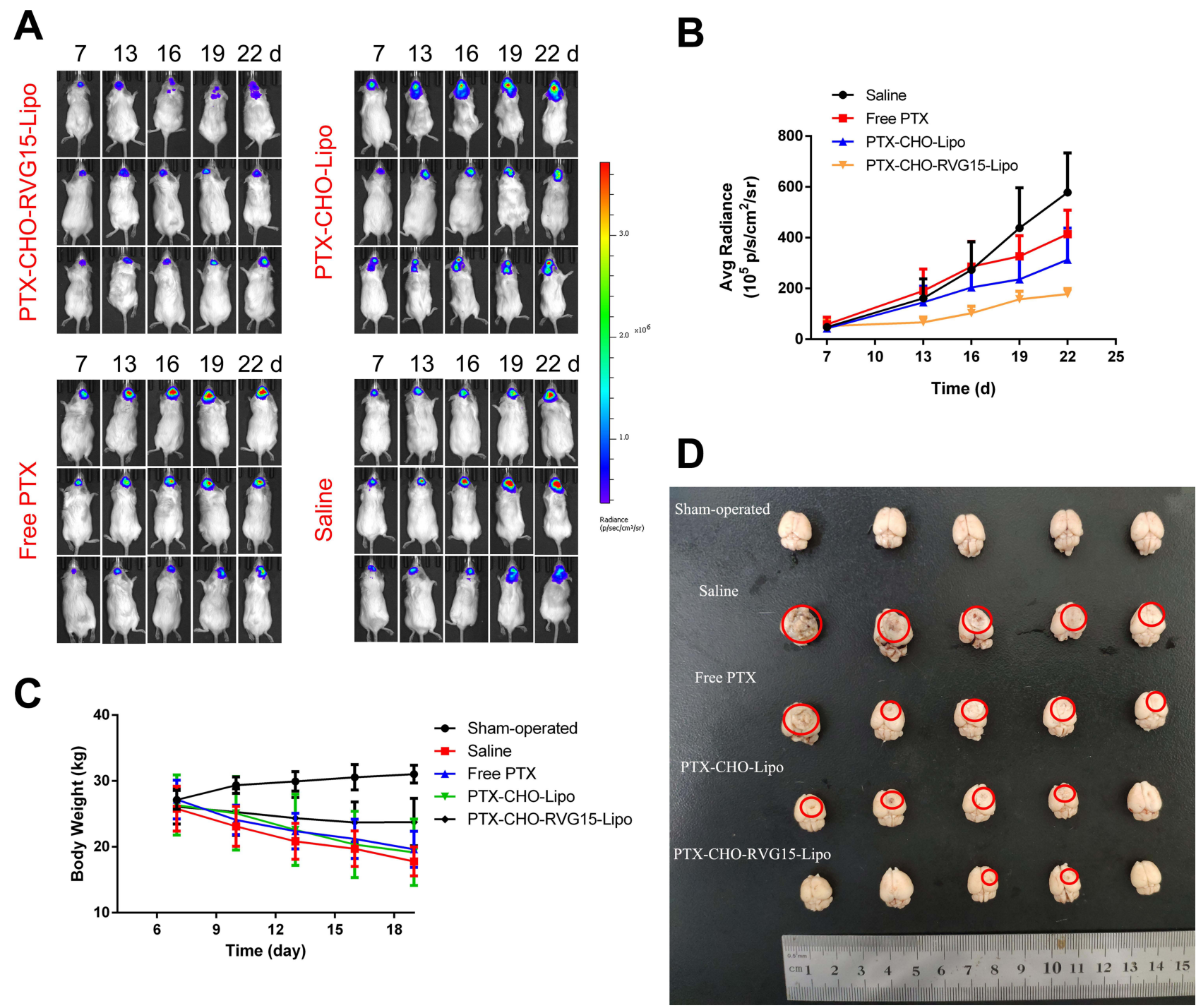

Figure 14 In vivo anti-glioma efficacy of PTX-CHO-RVGI5-Lipo in C6 $6^{\text {luc }}$ orthotopic glioma-bearing mice. PTX-CHO-Lipo, free PTX and saline are used as controls. The drug was given on day 7, 10, 13, 16, 19, 22 after tumor implantation (drug dosage: $7.5 \mathrm{mg}$ PTX equiv./kg).

Notes: $(\mathbf{A})$ Tumor growth in time monitored by bioluminescence imaging $(n=6)$. (B) Bioluminescence intensity changes $(n=6, M e a n s \pm S D)$. $(C)$ Body weight changes $(n=6)$. (D) Images of brain tissues of the mice.

Abbreviations: PTX, paclitaxel; PTX-CHO-Lipo, paclitaxel-cholesterol complex-loaded liposomes; PTX-CHO-RVG I5-Lipo, paclitaxel-cholesterol complex-loaded RVG I5modified liposomes.

all groups showed no significant hyperemia, necrosis, or inflammation in heart, spleen and kidney, indicating different PTX formulations had no obvious side effects.

Next, the effect of different preparations on survival rates was investigated. Figure 16 shows that PTX-CHORVG15-Lipo-treated group had the best anti-glioma activity, which prolonged the medium survival time to $50 \mathrm{~d}$, much longer than the other groups treated with saline (18.5 d), free PTX (20 d) and PTX-CHO-Lipo (33.5 d). The results demonstrated that the survival time of gliomabearing mice could be effectively prolonged by PTXCHO-RVG15-Lipo.
To sum up, RVG15-modified drug delivery system loading PTX displayed an outstanding therapeutic effect for brain tumors, including enhanced glioma inhibition, greater tumor cell apoptosis induction, less side effects, reduced metastasis and prolonged survival time through efficient BBB transport and deeper glioma penetration.

\section{Conclusion}

In this study, we developed an RVG15-modified liposomal drug delivery system with excellent physicochemical properties and evaluated the anti-glioma effect both in vitro and in vivo by constructing an orthotopic mouse 
A

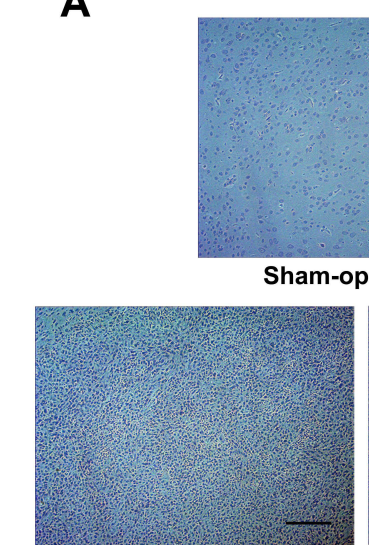

Saline

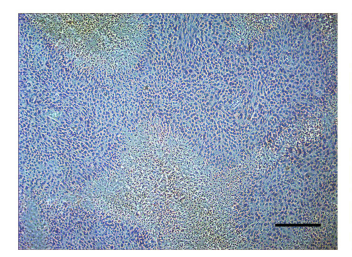

PTX-CHO-Lipo
B

Sham-operated

Saline

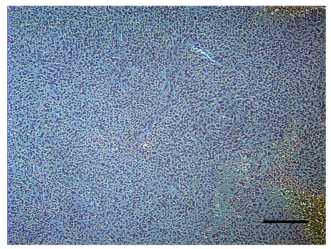

Free PTX

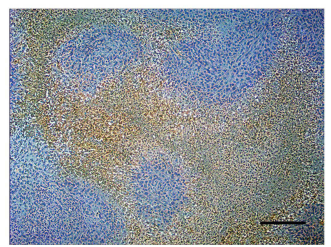

PTX-CHO-RVG15-Lipo

PTX-CHO-RVG15-Lipo

PTX-CHO-Lipo
Lung

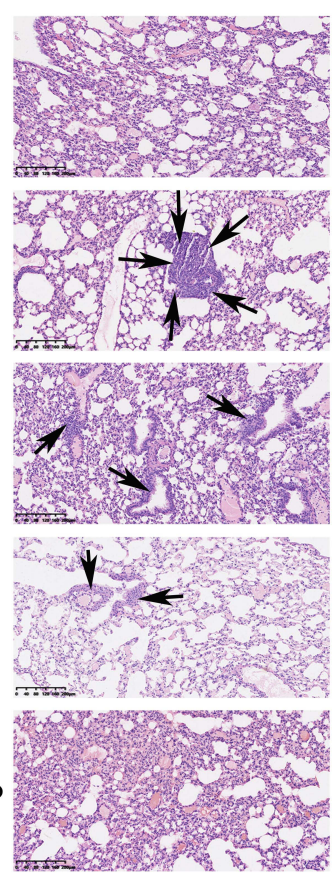

Liver
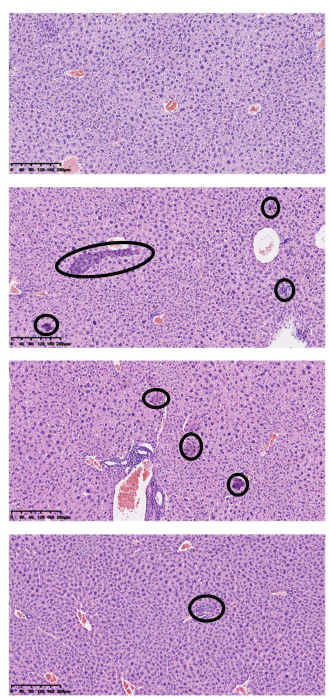

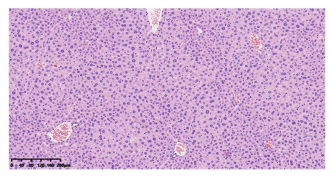

C

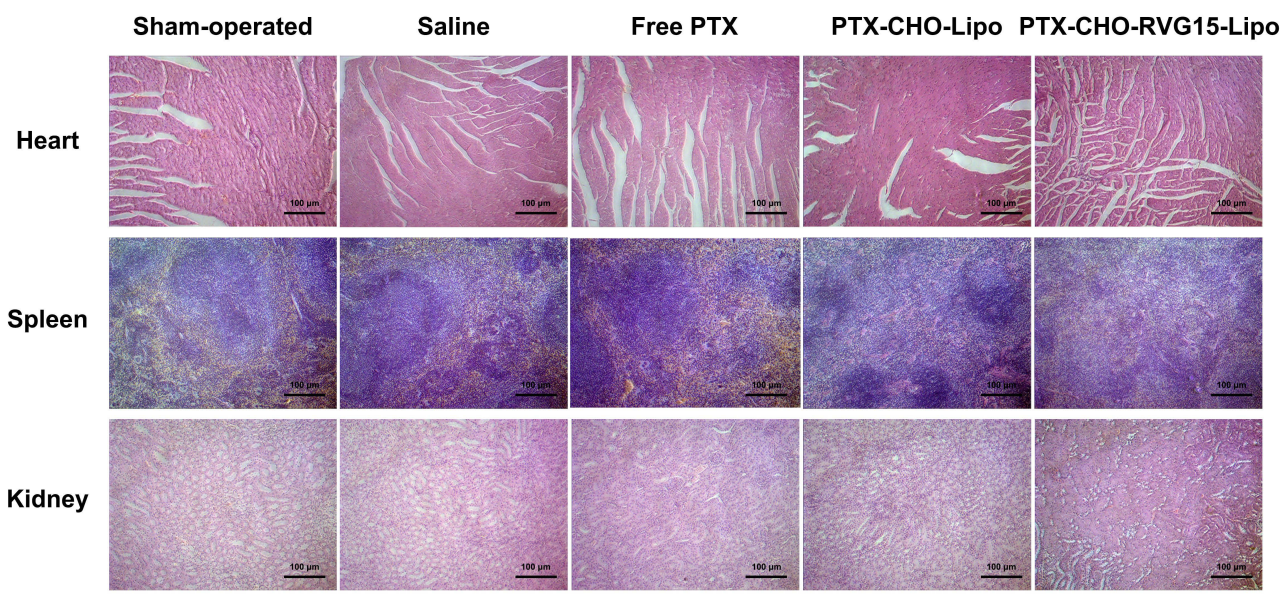

Figure 15 (A) TUNEL assay of tumor tissues isolated from mice treated with saline, free PTX, PTX-CHO-Lipo and PTX-CHO-RVG I5-Lipo observed by optical microscope (magnification $\times 100$ ). Scale bar: $100 \mu \mathrm{m}$. Brown areas showed apoptosis of tumor cells. (B) Lungs and livers of normal and glioma-bearing mice treated with saline, free PTX, PTX-CHO-Lipo and PTX-CHO-RVGI5-Lipo were stained with H\&E and observed by optical microscope (magnification $\times 100$ ). Scale bar: $200 \mu$ m. Black arrows and black circles showed the metastatic areas. (C) H\&E staining of heart, spleen and kidney after treatment of different PTX formulations observed by optical microscope (magnification $\times 100$ ). Scale bar: $100 \mu \mathrm{m}$.

Abbreviations: PTX, paclitaxel; PTX-CHO-Lipo, paclitaxel-cholesterol complex-loaded liposomes; PTX-CHO-RVG I5-Lipo, paclitaxel-cholesterol complex-loaded RVG I5modified liposomes.

glioma model. Firstly, we successfully synthesized RVG15-PEG 2000 -DSPE as a novel brain targeted material to improve the BBB penetration ability of nanocarrier, and meanwhile prepared the PTX-CHO complex to increase encapsulation efficiency and liposomes stability. Our designed nanocarrier (PTX-CHO-RVG15Lipo) based on RVG15-PEG 2000 -DSPE and PTX-CHO complex had been exhibited superior BBB transport efficiency, brain endothelial and glioma cell selectivity, glioma accumulation and deep penetrating capabilities. In addition, PTX-CHO-RVG15-Lipo demonstrated a considerable impact on the anti-proliferation and apoapsis of glioma cells in vitro. More importantly, PTX-CHO-RVG15-Lipo significantly inhibited tumor 


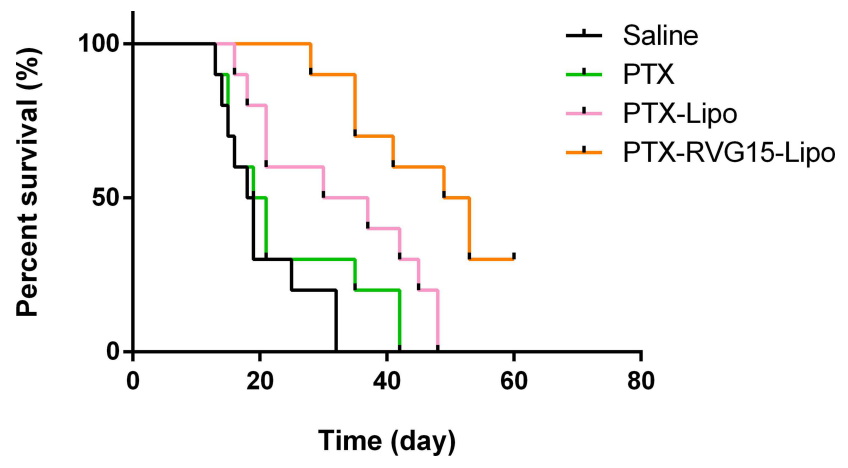

Figure 16 Kaplan-Meier survival curves of survival ratio of $\mathrm{C6}^{\text {luc }}$ glioma-bearing mice treated with saline, free PTX, PTX-CHO-Lipo, PTX-CHO-RVGI5-Lipo, respectively $(n=10)$.

Abbreviations: PTX, paclitaxel; PTX-CHO-Lipo, paclitaxel-cholesterol complexloaded liposomes; PTX-CHO-RVGI5-Lipo, paclitaxel-cholesterol complex-loaded RVGI5-modified liposomes.

growth and metastasis, and thus prolonged the survival life time of mouse with orthotopic glioma. Overall, these results demonstrated that the RVG15 peptide had great potential to be an effective ligand for BBB penetration and glioma targeting, and PTX-CHO-RVG15Lipo could serve as a novel drug delivery system for PTX and other chemotherapeutic agents in the field of glioma treatment.

\section{Acknowledgments}

This work was supported by National Natural Science Foundation of China (82073778), the Fundamental Research Funds for the Central Universities (3332021044, China), the Drug Innovation Major Project (2018ZX09711001-002-005, China), CAMS Innovation Fund for Medical Sciences (2019I2M-1-005, China) and Independent Project of State Key Laboratory of Bioactive Substance and Function of Natural Medicines (GTZA201808, China).

\section{Disclosure}

The authors report no conflicts of interest in this work.

\section{References}

1. Yang H, Wei L, Xun Y, Yang A, You H. BRD4: an emerging prospective therapeutic target in glioma. Mol Ther Oncolytics. 2021;21:1-14. doi:10.1016/j.omto.2021.03.005

2. Lin Z, Yang R, Li K, et al. Establishment of age group classification for risk stratification in glioma patients. Bmc Neurol. 2020;20(1):310. doi:10.1186/s12883-020-01888-w

3. Reni M, Mazza E, Zanon S, Gatta G, Vecht CJ. Central nervous system gliomas. Crit Rev Oncol Hematol. 2017;113:213-234. doi: 10.1016/j.critrevonc.2017.03.021
4. Tianqin G, Chunlei C, Jingjing W. Synergistic anti-glioma effects in vitro and in vivo of enediyne antibiotic neocarzinostatin and paclitaxel via enhanced growth delay and apoptosis-induction. Biol Pharm Bull. 2016;39(10):1623-1630. doi:10.1248/bpb.b16-00285

5. Cuddapah VA, Robel S, Watkins S, Sontheimer H. A neurocentric perspective on glioma invasion. Nat Rev Neurosci. 2014;15 (7):455-465. doi:10.1038/nrn3765

6. Pardridge WM. Drug targeting to the brain. Pharm Res. 2007;24 (9):1733-1744. doi:10.1007/s11095-007-9324-2

7. Gao X, Li C. Nanoprobes visualizing gliomas by crossing the blood brain tumor barrier. Small. 2014;10(3):426-440. doi:10.1002/ smll.201301673

8. Grabrucker AM, Ruozi B, Belletti D, et al. Nanoparticle transport across the blood brain barrier. Tissue Barriers. 2016;4(1):e1153568. doi:10.1080/21688370.2016.1153568

9. Serwer LP, James CD. Challenges in drug delivery to tumors of the central nervous system: an overview of pharmacological and surgical considerations. Adv Drug Deliv Rev. 2012;64(7):590-597. doi:10.1016/j.addr.2012.01.004

10. Gao H, Pang Z, Jiang X. Targeted delivery of nano-therapeutics for major disorders of the central nervous system. Pharm Res. 2013;30 (10):2485-2498. doi:10.1007/s11095-013-1122-4

11. Chen Y, Liu L. Modern methods for delivery of drugs across the blood-brain barrier. Adv Drug Deliv Rev. 2012;64(7):640-665. doi: 10.1016/j.addr.2011.11.010

12. Lu W, Xiong C, Zhang R, et al. Receptor-mediated transcytosis: a mechanism for active extravascular transport of nanoparticles in solid tumors. J Control Release. 2012;161(3):959-966. doi:10.1016/j. jconrel.2012.05.014

13. Vyas A, Jain A, Hurkat P, Jain A, Jain SK. Targeting of AIDS related encephalopathy using phenylalanine anchored lipidic nanocarrier. Colloids Surf B Biointerfaces. 2015;131:155-161. doi:10.1016/j. colsurfb.2015.04.049

14. Alvarez-Erviti L, Seow Y, Yin H, Betts C, Lakhal S, Wood MJ. Delivery of siRNA to the mouse brain by systemic injection of targeted exosomes. Nat Biotechnol. 2011;29(4):341-345. doi:10.1038/nbt.1807

15. Kumar P, Wu H, McBride JL, et al. Transvascular delivery of small interfering RNA to the central nervous system. Nature. 2007;448 (7149):39-43. doi:10.1038/nature05901

16. Lafon M. Rabies virus receptors. J Neurovirol. 2005;11(1):82-87. doi:10.1080/13550280590900427

17. Iskandar AR, Miao B, Li X, Hu KQ, Liu C, Wang XD. $\beta$ cryptoxanthin reduced lung tumor multiplicity and inhibited lung cancer cell motility by downregulating nicotinic acetylcholine receptor $\alpha 7$ signaling. Cancer Prev Res. 2016;9(11):875-886. doi:10.1158/ 1940-6207.CAPR-16-0161

18. Martinez AK, Jensen K, Hall C, et al. Nicotine promotes cholangiocarcinoma growth in xenograft mice. Am J Pathol. 2017;187 (5):1093-1105. doi:10.1016/j.ajpath.2017.01.011

19. Hua H, Zhang X, Mu H, et al. RVG29-modified docetaxel-loaded nanoparticles for brain-targeted glioma therapy. Int $J$ Pharm. 2018;543(1-2):179-189. doi:10.1016/j.ijpharm.2018.03.028

20. Park TE, Singh B, Li H, et al. Enhanced BBB permeability of osmotically active poly(mannitol-co-PEI) modified with rabies virus glycoprotein via selective stimulation of caveolar endocytosis for RNAi therapeutics in Alzheimer's disease. Biomaterials. 2015;38:61-71. doi:10.1016/j.biomaterials.2014.10.068

21. Son S, Hwang DW, Singha K, et al. RVG peptide tethered bioreducible polyethylenimine for gene delivery to brain. J Control Release. 2011;155(1):18-25. doi:10.1016/j.jconrel.2010.08.011

22. Liu Y, Huang R, Han L, et al. Brain-targeting gene delivery and cellular internalization mechanisms for modified rabies virus glycoprotein RVG29 nanoparticles. Biomaterials. 2009;30(25):4195-4202. doi:10.1016/j.biomaterials.2009.02.051 
23. Gotti C, Clementi F. Neuronal nicotinic receptors: from structure to pathology. Prog Neurobiol. 2004;74(6):363-396. doi:10.1016/j. pneurobio.2004.09.006

24. Han M, Xing H, Chen L. et al. Efficient antiglioblastoma therapy in mice through doxorubicin-loaded nanomicelles modified using a novel brain-targeted RVG-15 peptide. J Drug Target. 2021. doi:10.1080/1061186X.2021.1912053

25. Banerjee I, De K, Mukherjee D, et al. Paclitaxel-loaded solid lipid nanoparticles modified with Tyr-3-octreotide for enhanced anti-angiogenic and anti-glioma therapy. Acta Biomater. 2016;38:69-81. doi:10.1016/j.actbio.2016.04.026

26. Sharma S, Verma A, Pandey G, Mittapelly N, Mishra PR. Investigating the role of pluronic-g-cationic polyelectrolyte as functional stabilizer for nanocrystals: impact on paclitaxel oral bioavailability and tumor growth. Acta Biomater. 2015;26:169-183. doi:10.1016/j.actbio.2015.08.005

27. Ma Y, Yu S, Ni S, et al. Targeting strategies for enhancing paclitaxel specificity in chemotherapy. Front Cell Dev Biol. 2021;9:626910. doi:10.3389/fcell.2021.626910

28. Cai D, Gao W, He B, et al. Hydrophobic penetrating peptide PFVYLI-modified stealth liposomes for doxorubicin delivery in breast cancer therapy. Biomaterials. 2014;35(7):2283-2294. doi:10.1016/j.biomaterials.2013.11.088

29. Chu XY, Huang W, Wang YL, et al. Improving antitumor outcomes for palliative intratumoral injection therapy through lecithin- chitosan nanoparticles loading paclitaxel- cholesterol complex. Int J Nanomedicine. 2019;14:689-705. doi:10.2147/IJN.S188667

30. Wang M, Chen L, Huang W, et al. Improving the anti-keloid outcomes through liposomes loading paclitaxel-cholesterol complexes. Int J Nanomed. 2019;14:1385-1400. doi:10.2147/IJN.S195375

31. Singh B, Mehta G, Kumar R, Bhatia A, Ahuja N, Katare OP. Design, development and optimization of nimesulide-loaded liposomal systems for topical application. Curr Drug Deliv. 2005;2(2):143-153. doi:10.2174/1567201053585985
32. Meng L, Chu X, Xing H, et al. Improving glioblastoma therapeutic outcomes via doxorubicin-loaded nanomicelles modified with borneol. Int J Pharm. 2019;567:118485. doi:10.1016/j.ijpharm.2019.118485

33. Srinivasan B, Kolli AR, Esch MB, Abaci HE, Shuler ML, Hickman JJ. TEER measurement techniques for in vitro barrier model systems. J Lab Autom. 2015;20(2):107-126. doi:10.1177/ 2211068214561025

34. Shi H, Wang J, Dong F, Wang X, Li H, Hou Y. The effect of proteoglycans inhibited by RNA interference on metastatic characters of human salivary adenoid cystic carcinoma. BMC Cancer. 2009;9:456. doi:10.1186/1471-2407-9-456

35. Xia XJ, Guo RF, Liu YL, et al. Formulation, characterization and hypersensitivity evaluation of an intravenous emulsion loaded with a paclitaxel-cholesterol complex. Chem Pharm Bull. 2011;59 (3):321-326. doi:10.1248/cpb.59.321

36. Petros RA, DeSimone JM. Strategies in the design of nanoparticles for therapeutic applications. Nat Rev Drug Discov. 2010;9 (8):615-627. doi:10.1038/nrd2591

37. Khalil IA, Kogure K, Akita H, Harashima H. Uptake pathways and subsequent intracellular trafficking in nonviral gene delivery. Pharmacol Rev. 2006;58(1):32-45. doi:10.1124/pr.58.1.8

38. Kim JY, Choi WI, Kim YH, Tae G. Brain-targeted delivery of protein using chitosan- and RVG peptide-conjugated, pluronic-based nano-carrier. Biomaterials. 2013;34(4):1170-1178. doi:10.1016/j. biomaterials.2012.09.047

39. Wei X, Zhan C, Chen X, Hou J, Xie C, Lu W. Retro-inverso isomer of Angiopep-2: a stable d-peptide ligand inspires brain-targeted drug delivery. Mol Pharm. 2014;11(10):3261-3268. doi:10.1021/mp500086e

40. Guan J, Jiang Z, Wang M, et al. Short peptide-mediated brain-targeted drug delivery with enhanced immunocompatibility. Mol Pharm. 2019;16 (2):907-913. doi:10.1021/acs.molpharmaceut.8b01216

41. Yang T, Cui FD, Choi MK, et al. Enhanced solubility and stability of PEGylated liposomal paclitaxel: in vitro and in vivo evaluation. Int J Pharm. 2007;338(1-2):317-326. doi:10.1016/j.ijpharm.2007.02.011
International Journal of Nanomedicine

\section{Publish your work in this journal}

The International Journal of Nanomedicine is an international, peerreviewed journal focusing on the application of nanotechnology in diagnostics, therapeutics, and drug delivery systems throughout the biomedical field. This journal is indexed on PubMed Central, MedLine, CAS, SciSearch ${ }^{\mathbb{}}$, Current Contents ${ }^{\mathbb{R}} /$ Clinical Medicine, $^{-}$

\section{Dovepress}

Journal Citation Reports/Science Edition, EMBase, Scopus and the Elsevier Bibliographic databases. The manuscript management system is completely online and includes a very quick and fair peer-review system, which is all easy to use. Visit http://www.dovepress.com/ testimonials.php to read real quotes from published authors. 